\title{
Noncommutative Riemannian geometry and diffusion on ultrametric Cantor sets*
}

\author{
John Pearson and Jean Bellissard
}

\begin{abstract}
An analogue of the Riemannian Geometry for an ultrametric Cantor set $(C, d)$ is described using the tools of Noncommutative Geometry. Associated with $(C, d)$ is a weighted rooted tree, its Michon tree [29]. This tree allows to define a family of spectral triples $\left(\mathcal{C}_{\text {Lip }}(C), \mathscr{H}, D\right)$ using the $\ell^{2}$-space of its vertices, giving the Cantor set the structure of a noncommutative Riemannian manifold. Here $\ell_{\text {Lip }}(C)$ denotes the space of Lipschitz continuous functions on $(C, d)$. The family of spectral triples is indexed by the space of choice functions, which is shown to be the analogue of the sphere bundle of a Riemannian manifold. The Connes metric coming from the family of these spectral triples allows to recover the metric on $C$. The corresponding $\zeta$-function is shown to have abscissa of convergence, $s_{0}$, equal to the upper box dimension of $(C, d)$. Taking the residue at this singularity leads to the definition of a canonical probability measure on $C$, which in certain cases coincides with the Hausdorff measure at dimension $s_{0}$. This measure in turn induces a measure on the space of choices. Given a choice, the commutator of $D$ with a Lipschitz continuous function can be interpreted as a directional derivative. By integrating over all choices, this leads to the definition of an analogue of the Laplace-Beltrami operator. This operator has compact resolvent and generates a Markov semigroup which plays the role of a Brownian motion on $C$. This construction is applied to the simplest case, the triadic Cantor set, where: (i) the spectrum and the eigenfunctions of the Laplace-Beltrami operator are computed, (ii) the Weyl asymptotic formula is shown to hold with the dimension $s_{0}$, (iii) the corresponding Markov process is shown to have an anomalous diffusion with $\mathbb{E}\left(d\left(X_{t}, X_{t+\delta t}\right)^{2}\right) \simeq \delta t \ln (1 / \delta t)$ as $\delta t \downarrow 0$.
\end{abstract}

Mathematics Subject Classification (2000). 46L87, 47D07, 58B34, 53B21, 81R60.

Keywords. Cantor sets, ultrametric, quantized calculus, zeta-functions, fractal exponents, Laplace-Beltrami operator.

\section{Introduction}

The present work aims to define a Noncommutative Riemannian structure on an ultrametric Cantor set $(C, d)$. To this end, according to the theory developed by Connes [11], a spectral triple $(\mathcal{A}, \mathscr{H}, D)$ will be defined, namely $\mathcal{A} \subset \mathcal{C}(C)$ is

\footnotetext{
*Work supported in part by NSF grants DMS 06009565.
} 
a dense ${ }^{*}$-subalgebra of the $\mathrm{C}^{*}$-algebras of complex-valued continuous functions over $C, \mathscr{H}$ is a Hilbert space on which the algebra $\mathcal{C}(C)$ is represented and $D$, called the Dirac operator, is a selfadjoint operator on $\mathscr{H}$ with compact resolvent such that $[D, a] \in \mathscr{B}(\mathscr{H})$ whenever $a \in \mathcal{A}$. In the present work, the spectral triple is even. Namely there is an operator $\Gamma$ on $\mathscr{H}$ such that $\Gamma=\Gamma^{*}$ and $\Gamma^{2}=\mathbf{1}$ and such that $\Gamma$ commutes with the representatives of $\mathcal{A}$ and anticommutes with the Dirac operator.

The predominant view of this article is that Cantor sets should be treated as the boundary of a tree. Using an idea of Michon [29], an ultrametric Cantor set $(C, d)$ defines a weighted, rooted tree graph $\mathcal{T}=\mathcal{T}(C, d)$, with a boundary that is isometrically equivalent to $C$. Moreover, there is a one-to-one correspondence between a certain class of weighted, rooted trees and ultrametrics on a Cantor set. The tree graph then allows to define a Hilbert space and a Dirac operator which in turn defines a spectral triple for which $\mathcal{A}$ is the space of Lipschitz continuous functions on $(C, d)$. Conversely, the ultrametric can be recovered from the spectral triple.

The $\zeta$-function $\zeta(s)=\operatorname{Tr}\left(|D|^{-s}\right)$ of the Dirac operator is shown to be holomorphic in the domain $\operatorname{Re}(s)>s_{0}$, where $s_{0}$ is the upper box dimension of $(C, d)$. Following Connes [11], [10], the map $\mu(f)=\lim _{s \downarrow s_{0}} \operatorname{Tr}\left(f|D|^{-s}\right) / \operatorname{Tr}\left(|D|^{-s}\right)$ defines a probability measure on $C$. When $C$ is the attractor of a self-similar iterated function system, this measure can be identified with the Hausdorff measure corresponding to the box dimension $s_{0}$. On the new Hilbert space $L^{2}(C, d \mu)$ there is an operator $\Delta_{s}, s \in \mathbb{R}$, defined via the Dirichlet form

$$
\left\langle-\Delta_{s} f, g\right\rangle=\frac{1}{2} \int_{\Upsilon(C)} \operatorname{Tr}\left(|D|^{-s}\left[D, \pi_{\tau}(f)\right]^{*}\left[D, \pi_{\tau}(g)\right]\right) d \nu(\tau) .
$$

Here $\Upsilon(C)$ is the space of choices on $C$ and serves as the analogue for $C$ of the unit sphere bundle of a manifold. It will be shown that for all $s \in \mathbb{R}, \Delta_{s}$ is the generator of a Markovian semigroup on $L^{2}(C, d \mu)$, which can be seen as the analogue of a Brownian motion on $(C, d)$.

The present work grew out of the authors' desire to create a spectral triple for the transversal, $\Xi$, of an aperiodic Delone set of finite type [3], [2], [4]. In this case, the transversal is an ultrametric Cantor set, but it is not clear that $\Xi$ should be embeddable in $\mathbb{R}^{d}$ for any $d \in \mathbb{N}^{+}$. Therefore, it was necessary to create a spectral triple that was reliant on the intrinsic data of the Cantor set. The original idea came from a proposal by Alain Connes [11] (Chap. IV.3.e) for the triadic Cantor set. He also used this formalism to describe some properties of the Julia set [11]. In [28], Michel Lapidus proposed a program for applying the techniques of noncommutative geometry to fractals. More specifically, he was interested in creating spectral triples on fractals that would recapture both the geometric properties (i.e., fractal dimension, Hausdorff measure, etc. [15]) and the analytic properties (i.e., Laplacian [25]) of the fractal. Since Lapidus outlined his program, there have been spectral triples proposed for many different types of fractals. In [9], Lapidus and his coauthors were able to 
perform much of his program for the Sierpinski gasket. For the Cantor set, most of the work in this direction is based on the spectral triple proposed by Connes for the triadic Cantor set. Connes' spectral triple as well as many others based on his (e.g., [21], [22]) require the Cantor set to be embedded in $\mathbb{R}^{d}$ for some $d \in \mathbb{N}^{+}$. In another direction, Christensen and Ivan [8] have proposed spectral triples for abstract compact metric spaces by gluing together spectral triples associated with pairs of points. They are able to recover an equivalent metric with one of their spectral triples but could not recover precise geometric information about their original space. The spectral triple given in the present article extends much of the previous work by recovering the appropriate fractal geometric information intrinsically without the necessity of embedding in $\mathbb{R}^{d}$. More importantly, this spectral triple allows to construct an analogue of the Laplacian on the Cantor set and therefore an analogue of Brownian motion.

Diffusion on Cantor sets is not entirely new and has been studied in various contexts mostly as a non-Archimedean field [1], [14], [26]. Del Muto and Figà-Talamanca have generalized this in [13], [18] for locally compact ultrametric spaces, where the group of isometries is transitive and therefore allows to treat the Cantor set as an abelian group. In both cases, the construction of the diffusion relies heavily on the algebraic structure that is given to the space. A particular interest in these constructions has been taken by physicists for its potential applications in creating a $p$-adic based model of space-time based on $p$-adic differential operators [33]. One of the most used and simplest of these operators is the Vladimirov operator [33], which has been used as the analogue of the Laplacian. In the present article, it is shown that the algebraic structure is unnecessary for the construction of an appropriate Laplacian. In particular, the transversal of the Fibonacci tiling has only one nontrivial isometry and therefore has no obvious algebraic structure. In the treatment of the example of the triadic Cantor set in the present article, it is shown that the Vladimirov operator is related to the phase of the Dirac operator and consequently forgets the information provided by the metric. Moreover, an eigenbasis for $\Delta_{s}$ is constructed and shown to be the basis of Haar wavelets, thus recovering the result of [27] for the Vladimirov operator. In another area, Favre and Jonsson [17] have used an ultrametric tree to analyze singularities of algebraic varieties. They define a Laplacian, but the relation to the present construction is unclear. The present article extends the work to date by providing precise asymptotic estimates as $t \rightarrow 0$ for the Brownian motion on the Cantor set. More examples, including the case of the transversal of an aperiodic, repetitive Delone set of finite type, will be studied in future papers.

\section{Statement of main results}

This section presents a brief summary of the main results of this article. As stated in the introduction, the main viewpoint here is that Cantor sets should be treated as the boundary of a tree. 
A Cantor set $C$ is a totally disconnected, compact, metrizable space without isolated points. It is well known that such a set is homeomorphic to $C_{0}=\{0,1\}^{\mathrm{N}}$ [7]. Therefore, up to homeomorphism, the Cantor set is unique. However, adding a metric changes this prospect entirely. The structure of the tree allows to capture the additional information provided by the metric.

Definition 1. Let $C$ be a Cantor set. A metric on $C$ will be called regular if it defines a topology on $C$ for which $C$ is a Cantor set. A metric on $C$ is an ultrametric if $d(x, y) \leq \max \{d(x, z), d(z, y)\}$ for all $x, y, z \in C$.

In the remainder of the article, all metrics considered are regular.

Let now $(C, d)$ be a metric Cantor set such that $d$ is a regular ultrametric. As mentioned previously, Michon was able to show that every ultrametric gives a weighted, rooted tree such that the boundary of the tree, $\partial \mathcal{T}$, is isometric to $C$. The set of vertices and edges of its Michon tree $\mathcal{T}$ will be denoted by $\mathcal{V}, \mathcal{E}$. Any $v \in \mathcal{V}$ defines a clopen set $[v] \subset \partial \mathcal{T}$ which is the set of all infinite paths starting at the root that contain $v$. It is then possible to build an even spectral triple $(\mathcal{A}, \mathscr{H}, D)$. The *algebra $\mathcal{A}$ will be chosen as the space $\mathcal{A}=\mathcal{C}_{\text {Lip }}(C)$ of Lipschitz continuous complex-valued functions defined on $C$. From the noncommutative standpoint, it is important to note that this algebra is dense in the $\mathrm{C}^{*}$-algebra $\ell(C)$ of continuous functions on $C$ and is invariant by the holomorphic functional calculus [5]. In particular, this implies that the $K$-theory of $\mathcal{A}$ is the same as $\mathscr{C}(C)$. The Hilbert space $\mathscr{H}$ is given by $\mathscr{H}=\ell^{2}(\mathcal{V}) \otimes \mathbb{C}^{2}$ where $\mathcal{V}$ is the set of vertices of $\mathcal{T}$. The grading operator is the multiplication by $\Gamma=\mathbf{1} \otimes \sigma_{3}$ where $\sigma_{3}=\operatorname{diag}\{+1,-1\}$ is the third Pauli matrix. The Dirac operator $D$ is the operator defined by $D \psi(v)=(\operatorname{diam}[v])^{-1} \sigma_{1} \psi(v)$ where $\sigma_{1}$ is the first Pauli matrix (equal to +1 off the diagonal and 0 on the diagonal). To define the representation of the algebra $\mathcal{A}$ the notion of choice is needed.

Definition 2. Let $C$ be a Cantor set with a regular ultrametric $d$. A choice function is a map $\tau: \mathcal{V} \mapsto C \times C$ such that, if $v \in \mathcal{V}$ and if $\tau(v)=(x, y)$, then both $x, y$ are in $[v]$ and $d(x, y)=\operatorname{diam}[v]$. The set of choice functions on $C$ is denoted by $\Upsilon(C)$.

In what follows $\tau(v)=(x, y)$ will be written $x=\tau_{+}(v), y=\tau_{-}(v)$. Then the *-representation $\pi_{\tau}$ of $\mathcal{A}$ is given by $\pi_{\tau}(f) \psi(v)=\operatorname{diag}\left\{f\left(\tau_{+}(v)\right), f\left(\tau_{-}(v)\right)\right\} \psi(v)$. It is important to note that the Dirac operator is independent of the choice function. In the Noncommutative Riemannian structure, the space of choices $\Upsilon(C)$ will play the role of the unit sphere subbundle of the tangent bundle. In particular the basic element of intuition is that a choice function is the analogue of a vector field of unit vectors on a manifold. With this intuition in mind, then $\left[D, \pi_{\tau}(f)\right]$ represents the directional derivative of $f$ in the direction of $\tau$. On $\mathbb{R}^{d}$, a function $f \in \mathcal{C}^{\infty}\left(\mathbb{R}^{d}\right)$ is such that the gradient $\|\nabla f\|_{\infty}<1$ if and only if the directional derivative $\left\|\partial_{\vec{v}} f\right\|_{\infty}<1$ for every $\vec{v} \in \mathbb{R}$. Therefore, by this reasoning it is natural to expect that the metric 
can be recovered by using the Connes distance on functions $f \in \mathcal{C}(C)$ such that $\left\|\left[D, \pi_{\tau}(f)\right]\right\|<1$ for every $\tau \in \Upsilon(C)$. This is shown in the following result.

Theorem 1. Let $C$ be a Cantor set with a regular ultrametric $d$. Then $d$ coincides with the Connes distance $\rho$ defined by

$$
\rho(x, y):=\sup \left\{|f(x)-f(y)|: f \in \mathcal{C}_{\text {Lip }}(C), \sup _{\tau \in \Upsilon(C)}\left\|\left[D, \pi_{\tau}(f)\right]\right\| \leq 1\right\} .
$$

This result indicates that the spectral triple defined above is sufficient to recover the metric $d$ on $C$ whenever $d$ is a regular ultrametric and when all possible choice functions are taken into account. In fact, $\rho$ is the typical Connes distance with respect to the spectral triple obtained by summing over all possible choice functions.

Following the idea of Connes [10], [11], let $\zeta(s):=\operatorname{Tr}\left(|D|^{-s}\right)$ be the $\zeta$-function associated with the Dirac operator. It is known that there is $s_{0}>0$ (possibly infinite) such that $\zeta$ is holomorphic with respect to $s$ in a half-plane of the form $\operatorname{Re}(s)>s_{0}$ and that $\zeta$ is singular at $s_{0}$. Then $s_{0}$ is called its abscissa of convergence.

Let $\mathcal{T}$ be the tree corresponding to $(C, d)$. Let $\left\{\lambda_{k}\right\}_{k=1}^{\infty}$ be the set of all distinct $\operatorname{diam}([v])$ for $v \in \mathcal{V}$, labeled in decreasing order. Let $M_{n}$ be the maximum number of children for vertices with diameter at least $\lambda_{n}$. Then the next result is the following:

Theorem 2. If $\left(\log M_{n}\right) /\left(-\log \lambda_{n}\right) \rightarrow 0$ as $n \rightarrow \infty$, then $s_{0}$ coincides with the upper box dimension $\overline{\operatorname{dim}}_{B}(C)$ of $(C, d)$.

A special case of Theorem 2 is when there is a uniform bound on the number of children - this happens for the attractor of a self-similar iterated function system and the transversal of the Fibonacci tiling. In any case, the hypothesis says intuitively that the number of children can grow but it must be compensated for by a decrease in the size of the children.

At last, $(C, d)$ will be called $\zeta$-regular whenever the abscissa of convergence of the $\zeta$-function is finite, if $\lim _{s \downarrow s_{0}}\left(s-s_{0}\right) \operatorname{Tr}\left(|D|^{-s}\right)>0$ and if, for any $f \in \mathcal{A}$, the following limit exists:

$$
\mu(f)=\lim _{s \downarrow s_{0}} \frac{\operatorname{Tr}\left(|D|^{-s} \pi_{\tau}(f)\right)}{\operatorname{Tr}\left(|D|^{-s}\right)}
$$

Theorem 3. Let $C$ be a $\zeta$-regular Cantor set with a regular ultrametric $d$. Then the limit (1) is independent of the choice function $\tau$ and defines a probability measure on $C$.

In the simplest cases, the authors have checked that $\mu$ coincides with the Hausdorff measure associated with the upper box dimension. In particular this is true when $C$ is the attractor of a self-similar iterated function system satisfying the strong separability condition. Given the measure $\mu$ it is possible to construct various operators on 
$L^{2}(C, \mu)$. In order to do so, it is necessary to use $\mu$ to define a measure $v$ on the space of choices $\Upsilon(C)$. It is then interesting to try to find the analogue of the LaplaceBeltrami operator on a Riemannian manifold. Locally, the Laplace-Beltrami operator on a Riemannian manifold can be defined as the average of the square of the directional derivatives, $\left|\nabla_{\vec{v}} f\right|^{2}$, over the unit sphere of the tangent space. In the case of the Cantor set, the local basis is infinite and is given by choice functions. Therefore since there is no local Euclidean structure on $\Upsilon(C)$, it is natural to define the analogue of the Laplace-Beltrami operator by the following.

Theorem 4. Let $C$ be a $\zeta$-regular Cantor set with a regular ultrametric d. Then the measure $\mu$ coming from the $\zeta$-function induces a measure $v$ on the space of choices $\Upsilon(C)$. Moreover, for all $s \in \mathbb{R}$ there is a closable Dirichlet form on the Hilbert space $L^{2}(C, \mu)$ defined by

$$
Q_{s}(f, g):=\frac{1}{2} \int_{\Upsilon(C)} \operatorname{Tr}\left(|D|^{-s}\left[D, \pi_{\tau}(f)\right]^{*}\left[D, \pi_{\tau}(g)\right]\right) d \nu(\tau),
$$

with $\operatorname{Dom}\left(Q_{s}\right)$ a dense subspace of the real Hilbert space $L^{2}(C, \mu)$.

When $\operatorname{Supp}(\mu)=C$ then the classical theory of Dirichlet forms [19] associates to $Q_{s}$ a non-positive definite self-adjoint operator $\Delta_{s}$ on $L^{2}(C, \mu)$, which generates a Markovian semigroup. It will be shown in the case of the triadic Cantor set $C_{3}$ that for $s=s_{0}, \Delta_{s_{0}}$ plays the role of a Laplacian on $C_{3}$ in the sense that the Weyl asymptotic formula gives $\mathcal{N}(\lambda) \sim c_{0} \lambda^{s_{0} / 2}$. The Markovian semigroup associated to $\Delta_{s_{0}}$ defines a stochastic process $\left(X_{t}\right)_{t \geq 0}$ with values in $C_{3}$. However, somewhat unexpectedly this Brownian motion on $C_{3}$ satisfies $\mathbb{E}\left(d\left(X_{t_{0}}, X_{t_{0}+t}\right)^{2}\right) \sim c_{1} t \ln (1 / t)$ as $t \downarrow 0$. This surprising subdominant contribution by $\ln (1 / t)$ needs further investigation.

\section{Rooted trees}

3.1. Basic definitions. This section is a reminder about rooted trees (see [6]). A graph is a triple $G=\{\mathcal{V}, \mathcal{E}, \psi\}$ where $\mathcal{V}$ is a non-empty countable set with elements called vertices and $\mathcal{E}$ is a countable set with elements called edges. The function $\psi: \mathcal{E} \rightarrow \mathcal{V} \times \mathcal{V}$ is called the incidence function, which assigns to each edge a pair of not necessarily distinct vertices. If $\psi(e)=(v, w)$ or $\psi(e)=(w, v)$ then $e$ is said to link $v$ and $w$ or, equivalently, $v$ and $w$ are called incident. The degree $|v|$ of a vertex $v \in \mathcal{V}$ is the number of edges $e \in \mathcal{E}$ linking it to another vertex. A graph $G$ is simple whenever (i) there are no edges $e$ such that $\psi(e)=(v, v)$ for some $v \in \mathcal{V}$ and (ii) if $e, e^{\prime}$ are two edges linking the same vertices then $e=e^{\prime}$. In what follows only simple graphs will be considered.

A walk on a graph $G$ is a double sequence $\left\{\left(v_{0}, v_{1}, \ldots, v_{n-1}, v_{n}\right) ;\left(e_{1}, e_{2}, \ldots, e_{n}\right)\right\}$ (where $n$ is finite or infinite) of incident vertices and edges linking them such that 
$\psi\left(e_{i}\right)=\left(v_{i-1}, v_{i}\right)$ for all $i>0$. For a simple graph it is sufficient to specify the sequence of vertices. A step of the walk is a triple of the form $\left(v_{i-1}, v_{i}, e_{i}\right)$. The length of the walk is the number $n$ of steps making this walk. If the walk is finite, the first and the last vertices of the sequence are said to be linked by the walk. If $v$ is one of the vertices of the walk, then the latter is said to pass through $v$. A path is a walk with pairwise distinct vertices. The graph $G$ is connected if given any two vertices there is a finite path linking them.

A cycle is a finite walk with at least three steps, such that the first and the last vertices coincide and all other vertices are pairwise distinct. A tree is a connected graph with no cycle. A rooted tree is a pair $(\mathcal{T}, 0)$ where $\mathcal{T}$ is a tree and 0 is a vertex of $\mathcal{T}$ called the root. By abuse of notation, $\mathcal{T}$ will denote a rooted tree, and the root will be implicit. Since $\mathcal{T}$ is a tree, given any pair of distinct vertices there is one and only one path linking them. In particular, there is a unique path linking the root to a given vertex. So there is a one-to-one correspondence between the set of vertices and the set of finite paths starting at the root.

On a rooted tree there is a partial order defined by $v \succeq w$ if the path from the root to $w$ necessarily passes through $v$. Then $w$ is called a descendant of $v$ and this will also be written as $w \preceq v$, while $v$ will be called an ancestor of $w$. If, in addition, $v, w$ are incident, then $v$ is called the father of $w$ and $w$ is called a child of $v$. The height, ht $(v)$, of a vertex $v$ is the length of the unique path linking the root to $v$. Hence the root has height 0 , its children have height 1 and so on.

3.2. The boundary of a rooted tree. In this section $\mathcal{T}$ will denote an infinite rooted tree with root 0 . The set $\mathcal{V}$ of its vertices is endowed with the discrete topology. Since it is infinite it is certainly not compact. A compactification of the tree can be defined by considering the boundary $\partial \mathcal{T}$ of this tree defined as follows:

Definition 3. If $\mathcal{T}$ is a rooted tree, its boundary $\partial \mathcal{T}$ is the set of infinite paths starting at the root.

A vertex is dangling if it has no child. Hence the boundary ignores dangling vertices. In what follows, only trees with no dangling vertices will be considered. This implies among other things that every finite path can be extended to an infinite path.

Example 1. Let $T_{2}$ be the infinite binary rooted tree. That is $T_{2}$ is the tree with a root and such that every vertex has exactly two children. Since every vertex has two children, the edge linking it to one child will labeled by 0 and the other by 1 . Hence any finite path starting at the root, and therefore any vertex, is labeled by a finite sequence of 0 's and 1's. The root is given by the empty sequence. Thus $\mathcal{T}_{2}$ can be seen as the set of finite sequences of 0's and 1's. Consequently, $\partial \mathcal{T}_{2}=\{0,1\}^{\mathrm{N}}$. The 
map $\Theta:\left(\varepsilon_{n}\right)_{n \in \mathbb{N}} \mapsto \sum_{i=0}^{\infty} 2 \varepsilon_{i} 3^{-(i+1)}$ defines a one-to-one map from $\partial \mathcal{T}_{2}$ onto the classical triadic Cantor set.

Definition 4. Let $\mathcal{T}$ be a rooted tree. If $v$ is a vertex, $[v] \subset \partial \mathcal{T}$ denotes the set of infinite paths starting at the root and passing through $v$.

Proposition 1. Let $\mathcal{T}$ be a rooted tree with no dangling vertex. Then the set $\{[v]: v \in \mathcal{V}\}$ is a basis of open sets for a topology on the boundary of $\mathcal{T}$ for which $\partial \mathcal{T}$ is totally disconnected. For this topology $\partial \mathcal{T}$ is compact if and only if each vertex has at most a finite number of children. It has no isolated points if and only if each vertex has one descendant with at least two children.

The proof of this result is standard and will therefore be omitted and left to the reader.

Definition 5. A tree will be called Cantorian if it has a root, no dangling vertex and if each vertex has a finite number of children as well as a descendant with more than one child.

Remark 1. By Proposition 1 this definition is equivalent to $\partial \mathcal{T}$ is a Cantor set.

Various surgical operations on a tree lead to similar boundaries. The first operation is edge reduction. Namely if there is a path $\gamma$ linking $v$ to one of its descendant $w$ such that each vertex of this path distinct from $v, w$ has only one child, then the graph can be reduced by suppressing these vertices and replacing the path by one edge. Hence if $x \in \partial \mathcal{T}$ is any path passing through $v$ and $w$, it also automatically passes through all of the vertices of $\gamma$. Then it can also be reduced and the reduction operation gives a one-to-one mapping between the boundary of the initial tree and the boundary of the reduced one. In addition $[u]=[w]$ whenever $v \succeq u \succeq w$ and $u \neq w$, so that this mapping is actually an homeomorphism.

The opposite of edge reduction will be called edge extension. Namely any edge can be replaced by a finite path with same end points so that each internal vertex of the path has only one child.

There is also the notion of vertex extension. Namely if $v$ is a vertex with at least three children then one child will be called $v_{0}$ and the others $v_{1}, \ldots, v_{r}$. Then a new vertex $u$ is created as a child of $v$ having $v_{1}, \ldots, v_{r}$ as children. As before, this vertex extension does define also an homeomorphism between the corresponding boundaries. In particular this implies the following proposition which is one of many ways of showing that every Cantor set is homeomorphic to $\{0,1\}^{\mathbb{N}}$.

Proposition 2. Let $\mathcal{T}$ be a Cantorian tree. Then there is a map made of the product of a possibly infinite family of edge reductions, edge extensions and vertex extensions, mapping $\mathcal{T}$ onto the binary tree $\mathcal{T}_{2}$ and defining a homeomorphism of their boundaries. 
Definition 6. Let $\mathcal{T}$ be a Cantorian tree. If $A \subset \partial \mathcal{T}$ then a vertex $v$ is a common ancestor of $A$ if $A \subset[v]$. If $A$ has more than one point, its least common prefix (or 1.c.p.) is the smallest of its ancestors. If $A=\{x, y\}$ the least common prefix will be denoted by $x \wedge y$.

Proposition 3. Let $\mathcal{T}$ be a Cantorian tree. The l.c.p. of a subset $A \subset \partial \mathcal{T}$ with more than one point always exists and is unique.

The proof of this result is elementary and will be left to the reader.

\section{Michon's correspondence}

For the sake of the reader, this section recalls Michon's correspondence between regular ultrametrics on a Cantor set $C$, profinite structures on $C$, and weighted, rooted trees.

4.1. Ultrametrics and profinite structures. This section shows the correspondence between ultrametrics and profinite structures on $C$ [29]. Let $C$ be a Cantor set with regular metric $d$. Following [23], given $\varepsilon>0$ and $x, y \in C$ let an $\varepsilon$-chain be a sequence $x_{0}=x, x_{1}, \ldots x_{n-1}, x_{n}=y$ of points in $C$ such that $d\left(x_{i}, x_{i+1}\right)<\varepsilon$. This gives rise to an equivalence relation $\stackrel{\mathcal{E}}{\sim}$ by defining $x \stackrel{\mathcal{E}}{\sim} y$ if there is an $\varepsilon$-chain between them. In such a case, $[x]_{\varepsilon}$ will denote the equivalence class of $x \in C$. It is then possible to define the separation of $x$ and $y$ by $\delta(x, y):=\inf \{\varepsilon: x \stackrel{\mathcal{E}}{\sim} y\}$.

Proposition 4. Let $C$ be a Cantor set with regular metric $d$. Then the separation $\delta$ is a regular ultrametric on $C$ and is the largest dominated by $d$.

Proof. By [23] (Ch. 29.3), $\delta$ induces an ultrametric on the set of connected components. Since $C$ is totally disconnected, $\delta$ is an ultrametric on $C$. If $d(x, y)=\varepsilon$ then $x \stackrel{\mathcal{E}}{\sim} y$. Therefore, $\delta(x, y) \leq d(x, y)$. Now let $d^{\prime}$ be another ultrametric on $C$ such that $d^{\prime}(x, y) \leq d(x, y)$ for $x, y \in C$. Then for any $\varepsilon$-chain $x_{0}=x, \ldots, x_{n}=y$,

$$
\begin{aligned}
d^{\prime}(x, y) & \leq \max \left\{d^{\prime}\left(x_{i}, x_{i+1}\right): 0 \leq i \leq n-1\right\} \\
& \leq \max \left\{d\left(x_{i}, x_{i+1}\right): 0 \leq i \leq n-1\right\}<\varepsilon
\end{aligned}
$$

Thus, $d^{\prime} \leq \delta$. For a proof that $\delta$ is regular see [23].

It follows at once from the proposition that if $d$ is an ultrametric then $d=\delta$. From now on, it will be assumed that $C$ is a Cantor set with regular ultrametric $d$.

Definition 7. A profinite structure on a Cantor set $C$ is given by an increasing family $\left\{R_{\varepsilon}: \varepsilon \in \mathbb{R}^{+}\right\}$of equivalence relations on $C$ that satisfy the following properties: 
(i) Each relation $R_{\varepsilon}$ is open in $C \times C$, and $R_{\varepsilon}=C \times C$ for a certain $\varepsilon$.

(ii) The family is continuous on the left: $\bigcup_{\varepsilon^{\prime}<\varepsilon} R_{\mathcal{E}^{\prime}}=R_{\varepsilon}$.

(iii) $\bigcap_{\varepsilon \in \mathbb{R}}+R_{\varepsilon}=\Delta$ (the diagonal of $C \times C$ ).

Proposition 5. On a Cantor set $C$, there is a one-to-one correspondence between profinite structures and regular ultrametrics.

A proof of this result is given in the appendix.

4.2. Weighted, rooted trees. Using the results of the last section, it is now possible to show the connection between Cantorian trees and ultrametrics on a Cantor set.

Definition 8. Let $\mathcal{T}$ be an infinite rooted tree with no dangling vertex. A weight on $\mathcal{T}$ is a function $\epsilon: \mathcal{V} \rightarrow \mathbb{R}^{+}$that satisfies the following:

(i) If $v \succ v^{\prime}$ then $\epsilon(v)>\epsilon\left(v^{\prime}\right)$.

(ii) For an infinite path $v_{0} v_{1} \cdots \in \partial \mathcal{T}, \lim _{n \rightarrow \infty} \epsilon(v)=0$.

A rooted tree along with its weight function will be called a weighted, rooted tree.

As mentioned previously, there are various surgical operations on trees that lead to the same boundary. Given a tree $\mathcal{T}$, any vertex with only one child can be reduced by the process of edge reduction. The weight function is then the restriction of the original weight function. A tree for which every vertex has at least two children will be called reduced.

Proposition 6. On a Cantor set $C$, there is a one-to-one correspondence between regular ultrametrics and reduced, weighted, rooted Cantorian trees. Moreover given a regular ultrametric $d$, the boundary $\partial \mathcal{T}$ of the corresponding weighted, rooted Cantorian tree is isometric to $(C, d)$. The weight function $\epsilon$ for $\mathcal{T}$ is such that $\epsilon(v)=\operatorname{diam}_{d}([v])$.

A proof of this result is given in the appendix.

4.3. Embedding of ultrametric Cantor sets. A simple application of Michon's correspondence is given by the following.

Theorem 5. Let $C$ be a Cantor set with regular ultrametric $d$. Let $\mathcal{T}$ with weight $\epsilon$ be the corresponding reduced, weighted, rooted Cantorian tree. If $\mathcal{V}^{*}$ denotes all the vertices of $\mathcal{T}$ except for the root, then there exists an isometric embedding of $C$ into the real Hilbert space $\ell_{\mathbb{R}}^{2}\left(\mathcal{V}^{*}\right)$. 
Proof. Let $x \in C$ and let $v_{0} v_{1} \ldots$ be the infinite path corresponding to $x$. Let

$$
\Phi(x):=\sum_{n=0}^{\infty} \sqrt{\frac{\epsilon\left(v_{n}\right)^{2}-\epsilon\left(v_{n+1}\right)^{2}}{2}}\left|v_{n+1}\right\rangle,
$$

where $\left\{|v\rangle: v \in \mathcal{V}^{*}\right\}$ denotes the canonical basis of $\ell_{\mathbb{R}}^{2}\left(\mathcal{V}^{*}\right)$. If $v \neq v^{\prime}$, then $\left\langle v, v^{\prime}\right\rangle=0$. Therefore,

$$
\|\Phi(x)\|^{2}=\sum_{n=0}^{\infty} \frac{\epsilon\left(v_{n}\right)^{2}-\epsilon\left(v_{n+1}\right)^{2}}{2}=\frac{\epsilon\left(v_{0}\right)^{2}}{2}
$$

and $\Phi(x) \in \ell_{\mathbb{R}}^{2}\left(\mathcal{V}^{*}\right)$. Thus, $\Phi$ is well defined. Let $y \in C$ with $y \neq x$. If $w_{0} w_{1} \ldots$ is the infinite path corresponding to $y$ then there exists an $n_{0} \geq 0$ such that $w_{n} \neq v_{n}$ for $n>n_{0}$ and $w_{n}=v_{n}$ for $n \leq n_{0}$. Then $x \wedge y=v_{n_{0}}$ and $d(x, y)=\epsilon\left(v_{n_{0}}\right)$. Moreover,

$$
\Phi(x)-\Phi(y)=\sum_{n=n_{0}}^{\infty} \sqrt{\frac{\epsilon\left(v_{n}\right)^{2}-\epsilon\left(v_{n+1}\right)^{2}}{2}}\left|v_{n+1}\right\rangle-\sum_{n=n_{0}}^{\infty} \sqrt{\frac{\epsilon\left(w_{n}\right)^{2}-\epsilon\left(w_{n+1}\right)^{2}}{2}}\left|w_{n+1}\right\rangle
$$

and consequently

$$
\|\Phi(x)-\Phi(y)\|^{2}=\sum_{n=n_{0}}^{\infty} \frac{\epsilon\left(v_{n}\right)^{2}-\epsilon\left(v_{n+1}\right)^{2}}{2}+\sum_{n=n_{0}}^{\infty} \frac{\epsilon\left(w_{n}\right)^{2}-\epsilon\left(w_{n+1}\right)^{2}}{2}=\epsilon\left(v_{n_{0}}\right)^{2} .
$$

Since $\epsilon\left(v_{n_{0}}\right)=d(x, y)$, it follows that $\Phi$ is indeed an isometry.

\section{A spectral triple}

Given Michon's correspondence, it is now possible to construct a spectral triple on a Cantor set $C$ with regular ultrametric $d$.

\subsection{Construction of the spectral triple}

Definition 9. An odd spectral triple for an involutive algebra $\mathcal{A}$ is a triple $(\mathcal{A}, \mathscr{H}, D)$, where $\mathscr{H}$ is a Hilbert space on which $\mathcal{A}$ has a representation $\pi$ by bounded operators. $D$ is a self-adjoint operator on $\mathscr{H}$ with compact resolvent such that $[D, \pi(a)]$ is a bounded operator for all $a \in \mathcal{A}$.

An even spectral triple is an odd spectral triple along with a grading operator $\Gamma: \mathcal{H} \rightarrow \mathscr{H} . \Gamma$ is required to satisfy $\Gamma^{*}=\Gamma, \Gamma^{2}=1, \Gamma D=-D \Gamma$, and $\Gamma \pi(a)=\pi(a) \Gamma$ for all $a \in \mathcal{A}$

The algebra will be $\ell_{\text {Lip }}(C)$. Let $\mathcal{T}$ be the reduced, weighted, rooted Cantorian tree corresponding to the regular ultrametric $d$. Since $\mathcal{T}$ is Cantorian, the set of 
vertices $\mathcal{V}$ is countable. Let $\mathscr{H}:=\ell^{2}(\mathcal{V}) \otimes \mathbb{C}^{2} . D$ is the operator on $\mathscr{H}$ given by $D \psi(v):=(\operatorname{diam}(v))^{-1} \sigma_{1} \psi(v)$ where $\sigma_{1}$ is the first Pauli matrix. The grading operator is the multiplication by $\Gamma:=\mathbf{1} \otimes \sigma_{3}$ where $\sigma_{3}=\operatorname{diag}\{+1,-1\}$ is the third Pauli matrix. As mentioned earlier, to define a representation on $\mathcal{A}$ a choice function is required (see Definition 2). Let $\tau \in \Upsilon(C)$ be a choice function. Then the ${ }^{*}$-representation $\pi_{\tau}$ of $\mathcal{A}$ is given by $\pi_{\tau}(f) \psi(v)=\operatorname{diag}\left\{f\left(\tau_{+}(v)\right), f\left(\tau_{-}(v)\right)\right\} \psi(v)$.

Proposition 7. $\pi_{\tau}$ is a faithful ${ }^{*}$-representation of $\mathcal{C}(C)$ for all $\tau \in \Upsilon(C)$.

Proof. That $\pi_{\tau}$ is a *-representation is obvious. It is bounded since $f$ is continuous and $C$ is compact. Let $f, g \in \mathscr{C}(C)$ be such that $\pi_{\tau}(f)=\pi_{\tau}(g)$. Then $f\left(\tau_{+}(v)\right)=$ $g\left(\tau_{+}(v)\right)$ for all $v \in \mathcal{V}$. For $x \in C$, there exists $v_{0}, v_{1}, \ldots \in \mathcal{V}$ such that $x \in\left[v_{j}\right]$ and $\operatorname{diam}\left(\left[v_{j}\right]\right) \rightarrow 0$. Hence $f(x)=\lim _{j \rightarrow \infty} f\left(\tau_{+}\left(v_{j}\right)\right)=\lim _{j \rightarrow \infty} g\left(\tau_{+}\left(v_{j}\right)\right)=g(x)$. Thus $\pi_{\tau}$ is faithful.

Based on this proposition, $\pi_{\tau}$ is also a faithful representation on $\mathcal{C}_{\mathrm{Lip}}(C)$.

Proposition 8. $\left(\mathcal{L}_{\mathrm{Lip}}(C), \mathcal{H}, D, \Gamma\right)$ is an even spectral triple for all $\tau \in \Upsilon(C)$.

Proof. To show that $D$ is self-adjoint, let $\psi, \psi^{\prime} \in \mathscr{H}$. First $D$ is defined on the set $\mathscr{D}$ of $\psi \in \mathscr{H}$ such that $\sum_{v \in \mathcal{V}}(\operatorname{diam}([v]))^{-2}\langle\psi(v), \psi(v)\rangle_{\mathbb{C}^{2}}<\infty$. This is a dense subspace of $\mathscr{H}$ and by Riesz' theorem, the domain of the adjoint coincides with $\mathscr{D}$. Since $D$ is obviously symmetric, it is self-adjoint indeed. The definition of $D$ shows that the eigenvalues of its inverse are $\pm \operatorname{diam}([v])$ so that, since the diameters are decreasing along the tree, each eigenvalue has finite multiplicity and the only point of accumulation is 0 . So $D$ has compact resolvent.

Let $v \in \mathcal{V}$. Because diam $([v])=d\left(\tau_{+}(v), \tau_{-}(v)\right)$ and $\tau$ is a choice function, we have

$$
\left(\left[D, \pi_{\tau}(f)\right] \psi\right)(v)=\frac{f\left(\tau_{+}(v)\right)-f\left(\tau_{-}(v)\right)}{d\left(\tau_{+}(v), \tau_{-}(v)\right)}\left(\begin{array}{rr}
0 & -1 \\
1 & 0
\end{array}\right) \psi(v) .
$$

Since $f$ is Lipschitz, $\left\|\left[D, \pi_{\tau}(f)\right] \psi\right\|_{\mathscr{H}} \leq k\|\psi\|_{\mathscr{H}}$, where $k$ is the Lipschitz constant of $f$ and $\left[D, \pi_{\tau}(f)\right] \in \mathscr{B}(\mathcal{H})$.

The proof that $\Gamma^{*}=\Gamma, \Gamma^{2}=\Gamma, \Gamma D=-D \Gamma$ and $\Gamma \pi_{\tau}(f)=\pi_{\tau}(f) \Gamma$ for all $f \in \mathcal{C}_{\text {Lip }}(C)$ is straightforward.

5.2. The Connes distance: proof of Theorem 1. The spectral triple should be able to recover some of the structure of the original space $C$. Theorem 1 shows that it can recover the metric when all possible choice functions are taken into account.

Proof of Theorem 1. Let $x, y \in C$ and let $d_{x}: C \rightarrow C$ be given by $d_{x}(y)=d(x, y)$. Then $d_{x}$ is Lipschitz continuous. Let $\tau \in \Upsilon(C)$ and recall that this implies that 
$d\left(\tau_{+}(v), \tau_{-}(v)\right)=\operatorname{diam}(v)$. Then

$$
\begin{aligned}
\left\|\left[D, \pi_{\tau}\left(d_{x}\right)\right]\right\|_{\mathcal{B}(\mathscr{H})} & =\sup _{v \in \mathcal{V}}\left\{\frac{\left|d\left(x, \tau_{+}(v)\right)-d\left(x, \tau_{-}(v)\right)\right|}{d\left(\tau_{+}(v), \tau_{-}(v)\right)}\right\} \\
& \leq \sup _{v \in \mathcal{V}}\left\{\frac{d\left(\tau_{+}(v), \tau_{-}(v)\right)}{d\left(\tau_{+}(v), \tau_{-}(v)\right)}\right\}=1,
\end{aligned}
$$

where the inequality follows from the triangle inequality. Consequently,

$$
\sup _{\tau \in \Upsilon(C)}\left\{\left\|\left[D, \pi_{\tau}\left(d_{x}\right)\right]\right\|_{\mathcal{B}(\mathscr{H})}\right\} \leq 1
$$

and $\rho(x, y) \geq\left|d_{x}(x)-d_{x}(y)\right|=d(x, y)$.

For $x, y \in C$, let $v \in \mathcal{V}$ be such that $v=x \wedge y$, so that $d(x, y)=\operatorname{diam}(v)$. Let $\tau$ be such that $\tau_{+}(v)=x$ and $\tau_{-}(v)=y$. Then

$$
\frac{\left|f\left(\tau_{+}(v)\right)-f\left(\tau_{-}(v)\right)\right|}{\operatorname{diam}(v)}=\frac{\left|f\left(\tau_{+}(v)\right)-f\left(\tau_{-}(v)\right)\right|}{d\left(\tau_{+}(v), d\left(\tau_{-}(v)\right)\right.} \leq 1
$$

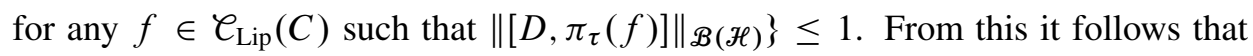
$|f(x)-f(y)| \leq d(x, y)$ and so $\rho(x, y) \leq d(x, y)$.

\section{6. $\zeta$-functions}

In this section the Dirac operator $D$ is used to create a $\zeta$-function as formulated by Connes [11]. Since the Dirac operator is independent of choice, this $\zeta$-function will also be independent of choice.

6.1. The $\zeta$-function for $D$. Let $\mathscr{H}$ be the Hilbert space from the previously created spectral triple. Then for $\psi \in \mathscr{H},(|D| \psi)(v)=\operatorname{diam}^{-1}(v) \psi(v)$. Since $\operatorname{diam}(v)>0$ for all $v \in \mathcal{V}$, it follows that $|D|$ is invertible and $\left(|D|^{-1} \psi\right)(v)=\operatorname{diam}(v) \psi(v)$. Let

$$
\zeta(s):=\frac{1}{2} \operatorname{Tr}\left(|D|^{-s}\right)=\sum_{v \in \mathcal{V}} \operatorname{diam}(v)^{s} .
$$

Then $\zeta$ is a Dirichlet series. By [24] (Ch. 2), as a function of the complex variable $s, \zeta$ either converges everywhere, nowhere, or in a half-plane given by $\operatorname{Re}(s)>s_{0}$. In the last case, $s_{0}$ is called the abscissa of convergence. Since the eigenvalues of $|D|^{-1}$ are discrete, put $\zeta(s)=\sum a_{k} \lambda_{k}^{s}$, where $\lambda_{1}=\operatorname{diam}(C)>\lambda_{2}>\cdots$ and $a_{k}$ is the multiplicity of $\lambda_{k}$, that is, the number of $v \in \mathcal{V}$ with diameter $\lambda_{k}$.

The following classical lemma on Dirichlet series will be useful.

Lemma 1 (Dirichlet). Let $\zeta(s)=\sum a_{k} \lambda_{k}^{s}$ be a Dirichlet series with abscissa of convergence $s_{0}$. Suppose further that all the $\lambda_{k}$ are positive, $\lambda_{1}>\lambda_{2}>\cdots$, and that $a_{k}>0$ for all $k$. Then

$$
\limsup _{k \rightarrow \infty} \frac{\log \sum_{j=1}^{j=k} a_{j}}{-\log \lambda_{k}}=s_{0}
$$


A proof of this can be found in [24] (Ch. 2.6). Note that the form of the Dirichlet series used there is slightly different than the one used here.

6.2. The upper box dimension. This section is a reminder about the upper box dimension of a fractal. For a treatment of the many fractal dimensions, the reader can consult [15]. Let $X$ be a metric space with metric $d$. Let $N_{\delta}(X)$ be the least number of sets of diameter at most $\delta$ that cover $X$.

Definition 10. The upper box dimension is defined as

$$
\overline{\operatorname{dim}}_{B}(C)=\limsup _{\delta \downarrow 0} \frac{\log N_{\delta}(C)}{-\log \delta}
$$

As shown in [15] (Ch. 2.1), the upper box dimension satisfies the following dimension properties: monotonicity, zero on finite sets, and it gives dimension $n$ to open sets in $\mathbb{R}^{n}$. Most importantly, it is invariant under bi-Lipschitz transformations. Therefore, if two different metrics on $X$ are metrically equivalent, then they have the same upper box dimension. The upper box dimension is also the largest of the typical fractal dimensions. In particular, it is greater than or equal to the Hausdorff dimension of $X$.

6.3. Proof of Theorem 2. As already defined, let $\left\{\lambda_{k}\right\}_{k=1}^{\infty}$ be the set of all distinct $\operatorname{diam}([v])$ for $v \in \mathcal{V}$, labeled in decreasing order. $M_{n}$ be the maximum number of children for vertices with diameter at least $\lambda_{n}$. In particular $M_{n+1} \geq M_{n}$.

For any $\delta>0$ such that $\lambda_{n}>\delta \geq \lambda_{n+1}$, we have $N_{\delta}(C)=N_{\lambda_{n+1}}(C)$ because there are no vertices with $\delta \geq \operatorname{diam}([v])>\lambda_{n+1}$. Thus,

$$
\frac{\log N_{\lambda_{n+1}}(C)}{-\log \lambda_{n+1}} \leq \frac{\log N_{\delta}(C)}{-\log \delta}<\frac{\log N_{\lambda_{n+1}}(C)}{-\log \lambda_{n}} .
$$

A minimal cover of $C$ with sets of diameter at most $\lambda_{n}$ must use every vertex of diameter $\lambda_{n}$. Thus, a cover of $C$ with sets of diameter at most $\lambda_{n+1}$ can be obtained from this cover by taking the children of each set of diameter $\lambda_{n}$ and keeping the other sets the same. This cover, $\mathcal{O}$, is in fact minimal since no $O \in \mathcal{O}$ can cover two children of a vertex of diameter $\lambda_{n}$. Since every vertex of diameter at least $\lambda_{n}$ has at least 2 children and at most $M_{n}$ children, this gives

$$
N_{\lambda_{n}}+a_{n} \leq N_{\lambda_{n+1}} \leq N_{\lambda_{n}}+\left(M_{n}-1\right) a_{n}
$$

After iterating the procedure,

$$
1+\sum_{k=1}^{n} a_{k} \leq N_{\lambda_{n+1}} \leq 1+\sum_{k=1}^{n}\left(M_{k}-1\right) a_{k} \leq 1+\left(M_{n}-1\right) \sum_{k=1}^{n} a_{k},
$$


where the 1 comes from the fact that $N_{\lambda_{1}}=1$. For the binary tree, it is easy to check that these inequalities are in fact equalities and therefore that this estimate is in some sense optimal. Since every cover of $C$ with sets of diameter at most $\lambda_{n+1}$ must use every vertex of diameter $\lambda_{n+1}$, it follows that $N_{\lambda_{n+1}} \geq a_{n+1}$. Consequently, $N_{\lambda_{n+1}} \geq 1 / 2\left(a_{n+1}+1+\sum_{k=1}^{n} a_{k}\right)$. Thus,

$$
\frac{\log 1 / 2\left(\sum_{k=1}^{k=n+1} a_{k}\right)}{-\log \lambda_{n+1}} \leq \frac{\log N_{\delta}(C)}{-\log \delta}<\frac{\log \left(1+\left(M_{n}-1\right) \sum_{k=1}^{k=n} a_{k}\right)}{-\log \lambda_{n}} .
$$

Therefore, since $\left(\log \left(M_{n}-1\right)\right) /\left(-\log \lambda_{n}\right) \rightarrow 0$ as $n \rightarrow \infty$, we have

$$
\limsup _{n \rightarrow \infty} \frac{\log \sum_{k=1}^{k=n+1} a_{k}}{-\log \lambda_{n+1}} \leq \limsup _{\delta \rightarrow 0} \frac{\log N_{\delta}(C)}{-\log \delta} \leq \limsup _{n \rightarrow \infty} \frac{\log \sum_{k=1}^{k=n} a_{k}}{-\log \lambda_{n}}
$$

Due to the Dirichlet Lemma 1, it follows that $\overline{\operatorname{dim}}_{B}(C)=s_{0}$.

\section{Measure theory on $C$}

This section extends the study of the noncommutative geometry of a Cantor set $C$ by studying a measure $\mu$ that is naturally defined on $C$.

7.1. $\zeta$-regularity: proof of Theorem 3. In order to study more deeply the geometry of $C$ it is necessary to make some assumptions on $C$.

Definition 11. A Cantor set $C$ with regular ultrametric $d$ is $\zeta$-regular if the abscissa of convergence, $s_{0}$, of its $\zeta$-function is finite and if, for any $f \in \varphi(C)$ and any $\tau \in \Upsilon(C)$,

$$
\lim _{s \downarrow s_{0}}\left(s-s_{0}\right) \operatorname{Tr}\left(|D|^{-s} \pi_{\tau}(f)\right)
$$

exists.

Given a $\zeta$-regular Cantor set and a choice function $\tau \in \Upsilon(C)$, it is then possible to define a measure $\mu_{\tau}$ on $C$ given by

$$
\mu_{\tau}(f)=\int_{C} f d \mu_{\tau}=\lim _{s \downarrow s_{0}} \frac{\operatorname{Tr}\left(|D|^{-s} \pi_{\tau}(f)\right)}{\operatorname{Tr}\left(|D|^{-s}\right)}
$$

Proof of Theorem 3. Let $\tau, \tau^{\prime} \in \Upsilon(C)$ and $f \in \ell_{\text {Lip }}(C)$ with Lipschitz constant $k$. For $\operatorname{Re}(s)>s_{0}$, since $|D|^{-s}$ and $\pi_{\tau}(f)$ is bounded, it follows that $|D|^{-s} \pi_{\tau}(f)$ is 
trace class and similarly $|D|^{-s} \pi_{\tau^{\prime}}(f)$ is trace class. Therefore,

$$
\begin{aligned}
\left|\operatorname{Tr}\left(|D|^{-s}\left(\pi_{\tau}(f)-\pi_{\tau^{\prime}}(f)\right)\right)\right| \leq & \sum_{v \in \mathcal{V}}\left|f\left(\tau_{+}(v)\right)-f\left(\tau_{+}^{\prime}(v)\right)\right| \operatorname{diam}(v)^{\operatorname{Re}(s)} \\
& +\sum_{v \in \mathcal{V}}\left|f\left(\tau_{-}(v)\right)-f\left(\tau_{-}^{\prime}(v)\right)\right| \operatorname{diam}(v)^{\operatorname{Re}(s)} \\
\leq & 2 \sum_{v \in \mathcal{V}} k \operatorname{diam}(v)^{\operatorname{Re}(s)+1} .
\end{aligned}
$$

Consequently,

$$
\left|\mu_{\tau}(f)-\mu_{\tau^{\prime}}(f)\right|=\left|\lim _{s \downarrow s_{0}} \frac{\operatorname{Tr}\left(|D|^{-s} \pi_{\tau}(f)\right)-\operatorname{Tr}\left(|D|^{-s} \pi_{\tau^{\prime}}(f)\right)}{\operatorname{Tr}\left(|D|^{-s}\right)}\right|=0
$$

since $\operatorname{Tr}\left(|D|^{-s_{0}-1}\right)<\infty$. Because $\ell_{\text {Lip }}(C)$ is dense in $\mathcal{C}(C)$ and $\pi_{\tau}$ is continuous for all $\tau \in \Upsilon(C)$, we have that $\mu_{\tau}$ and $\mu_{\tau^{\prime}}$ are equal on $\mathcal{C}(C)$. Since $\pi_{\tau}$ is faithful for all $\tau \in \Upsilon(C)$, it follows that $\mu_{\tau}$ is a probability measure for each $\tau$.

7.2. The measure on the space of choices. In what follows, it will be necessary to have a measure on the spaces of choices, $\Upsilon(C)$. Recall that $\Upsilon(C)$ was the set of all functions $\tau: \mathcal{V} \rightarrow C \times C$ such that $\tau(v) \in[v] \times[v]$ and $d\left(\tau_{+}(v), \tau_{-}(v)\right)=\operatorname{diam}(v)$. Let $\mathcal{Y} \subset \mathcal{V} \times \mathcal{V}$ be defined to be the set of all brothers. That is, $(u, v) \in \mathcal{G}$ if $u$ and $v$ have the same parent and $u \neq v$. Let $\boldsymbol{E}_{v}$ be the set of all brothers whose parent is $v$. Now $x, y \in[v]$ are such that $d(x, y)=\operatorname{diam}([v])$ if and only if there is a unique pair $\left(w, w^{\prime}\right) \in \mathscr{G}_{v}$ of distinct children of $v$ such that $x \in[w]$ and $y \in\left[w^{\prime}\right]$. Consequently

$$
\Upsilon(C)=\prod_{v \in \mathcal{V}} \bigsqcup_{\left(w, w^{\prime}\right) \in \mathscr{E}_{v}}[w] \times\left[w^{\prime}\right] .
$$

Therefore, define a measure $v_{v}$ on $\Upsilon_{v}(C):=\bigsqcup_{\left(w, w^{\prime}\right) \in \mathscr{E}_{v}}[w] \times\left[w^{\prime}\right]$ by

$$
v_{v}=\frac{\mu \times \mu}{\sum_{\left(w, w^{\prime}\right) \in \mathscr{E}_{v}} \mu([w]) \mu\left(\left[w^{\prime}\right]\right)} .
$$

This is then a probability measure on $\Upsilon_{v}(C)$. Using the Kolmogorov Consistency Theorem [30] (V.5), there is an extension of these measures to a probability measure $v$ on $\Upsilon(C)$. This measure $v$ is such that $v\left(\left(\prod_{w \neq v} \Upsilon_{w}(C)\right) \times U_{v}\right)=v_{v}\left(U_{v}\right)$ for any $v_{v}$-measurable set $U_{v}$.

\section{Dirichlet forms and the operator $\Delta$}

In this section let $\mathscr{L}_{\mathbb{C}}^{2}(C, d \mu)$ denote the Hilbert space completion of $\mathcal{C}(C, \mathbb{C})$ with respect to $\langle f, g\rangle=\int_{C} \bar{f} g d \mu$ and let $\mathscr{L}^{2}(C, d \mu)$ denote the Hilbert space completion of $\mathcal{C}(C, \mathbb{R})$ with respect to the same inner product. It is of interest to study Markovian semigroups of operators on $\mathscr{L}^{2}(C, d \mu)$. As shown in [19], the study of Markovian semigroups is equivalent to studying the Dirichlet forms on $\mathscr{L}^{2}(C, d \mu)$. 
8.1. Dirichlet forms: proof of Theorem 4. Given a real Hilbert space $\mathscr{H}$, a nonnegative definite symmetric bilinear form densely defined on $\mathscr{H}$ is called a symmetric form on $\mathscr{H}$. Let $Q$ be a symmetric form on a Hilbert space $H$. If $\operatorname{Dom}(Q)$ is complete with respect to the metric given by $\langle f, g\rangle_{1}=\langle f, g\rangle_{H}+Q(f, g)$, where $\langle\cdot, \cdot\rangle_{H}$ is the inner product on $H$, then $Q$ is called a closed form. Given a closed symmetric form $Q$ on $\mathscr{L}^{2}(C, d \mu)$, then $Q$ is called Markovian if $Q(\tilde{f}, \tilde{f}) \leq Q(f, f)$ where $\tilde{f}=\min (\max (0, f), 1)$. If $Q$ is not closed, the condition to be Markovian is more complicated; however, the previous condition is sufficient. A closed symmetric Markovian form is called a (symmetric) Dirichlet form. Given the formalism of the previous sections, it is possible to define a form $Q_{s}$ on $\mathscr{L}_{\mathbb{C}}^{2}(C, d \mu)$ by

$$
Q_{s}(f, g):=\frac{1}{2} \int_{\Upsilon(C)} \operatorname{Tr}\left(|D|^{-s}\left[D, \pi_{\tau}(f)\right]^{*}\left[D, \pi_{\tau}(g)\right]\right) d \nu(\tau) .
$$

It is now necessary to specify a domain for the form. Let $\mathscr{E} \subset \mathscr{L}^{2}(C, d \mu)$ be the real linear space spanned by $\left\{\chi_{v}: v \in \mathcal{V}\right\}$, where $\chi_{v}$ is the characteristic function of $[v] \subset C$.

Lemma 2. $\&$ is dense in $\mathscr{L}^{2}(C, d \mu)$.

Proof. Let $f \in \mathcal{C}(C)$. Since $f$ is continuous and $C$ is compact, $f$ is uniformly continuous. Consequently, for $\varepsilon>0$ there is a $\delta>0$ such that if $d(x, y)<\delta$ then $|f(x)-f(y)|<\varepsilon$. Let $v_{1}, \ldots, v_{N}$ be a partition of $C$ such that $\operatorname{diam}\left(\left[v_{i}\right]\right)<\delta$. Let $\tau \in \Upsilon(C)$. Let $g$ be defined by $g(x):=f\left(\tau_{+}\left(v_{j}\right)\right)$, where $v_{j}$ is the unique vertex of the partition such that $x \in\left[v_{j}\right]$. Then $\|f-g\|_{\infty}<\varepsilon$ and consequently $\|f-g\|_{2}<\varepsilon$. Thus $\mathcal{E}$ is dense in $\ell(C)$. Then since $\mathscr{C}(C)$ is dense in $\mathscr{L}^{2}(C, d \mu)$, so is $\mathscr{E}$.

Let $\operatorname{Dom}\left(Q_{s}\right)=\varepsilon$.

Proof of Theorem 4. It is clear that $Q_{s}$ must be bilinear. It is symmetric because of the trace and because $|D|^{-s}$ commutes with $\left[D, \pi_{\tau}(f)\right]$ for all $f \in \mathcal{C}_{\text {Lip }}(C)$. Now

$$
\left[D, \pi_{\tau}(f)\right]^{*}\left[D, \pi_{\tau}(g)\right] \psi(v)=\frac{f\left(\tau_{+}(v)\right)-f\left(\tau_{-}(v)\right)}{\operatorname{diam}(v)} \frac{g\left(\tau_{+}(v)\right)-g\left(\tau_{-}(v)\right)}{\operatorname{diam}(v)} \psi(v)
$$

and thus

$$
\operatorname{Tr}\left(|D|^{-s}\left[D, \pi_{\tau}(f)\right]^{*}\left[D, \pi_{\tau}(f)\right]\right)=2 \sum_{v \in \mathcal{V}} \operatorname{diam}(v)^{s-2}\left(f\left(\tau_{+}(v)\right)-f\left(\tau_{-}(v)\right)\right)^{2} .
$$

Consequently, $Q_{s}$ is non-negative definite. Since $\chi_{v}\left(\tau_{+}(w)\right)-\chi_{v}\left(\tau_{-}(w)\right)=0$ if $w \nsucc v$, it follows that $\left[D, \pi_{\tau}\left(\chi_{v}\right)\right]$ is of finite rank for each characteristic function $\chi_{v}$ with $v \in \mathcal{V}$. Thus, for $f \in \mathcal{E},\left[D, \pi_{\tau}(f)\right]$ is of finite rank and $Q_{s}(f, g)<\infty$ for all $g \in \mathscr{L}^{2}(C, d \mu)$. 
Let now $\left(f_{n}\right)_{n \in \mathbb{N}}$ be a sequence of functions in $\mathscr{E}$ such that $\lim _{n \rightarrow \infty}\left\|f_{n}\right\|_{\mathscr{L}^{2}}=0$ and $\lim _{n, m \rightarrow \infty} Q_{s}\left(f_{n}-f_{m}, f_{n}-f_{m}\right)=0$. To show that $Q_{s}$ is closable, it is then necessary to show that $\lim _{n \rightarrow \infty} Q_{s}\left(f_{n}, f_{n}\right)=0$. Since $\lim _{n \rightarrow \infty}\left\|f_{n}\right\|_{\mathscr{L}^{2}}=0$, there is a subsequence $f_{n_{i}}$ that converges pointwise $\mu$-a.e. to 0 [31] (Thm. 3.12). In particular, due to the definition of the measure $v$ on the set of choices, $f_{n_{i}}\left(\tau_{+}(v)\right) \rightarrow 0$ for $v$-a.e. choice and for all $v \in \mathcal{V}$. Similarly for $\tau_{-}(v)$. So, given $\varepsilon>0$ let $N$ be such that $Q_{s}\left(f_{n}-f_{m}, f_{n}-f_{m}\right)<\varepsilon$ for $n, m>N$. Then for $m>N$,

$$
Q_{s}\left(f_{m}, f_{m}\right)=\int_{\Upsilon(C)} \sum_{j=1}^{K} \operatorname{diam}\left(v_{j}\right)^{s-2}\left(f_{m}\left(\tau_{+}\left(v_{j}\right)\right)-f_{m}\left(\tau_{-}\left(v_{j}\right)\right)\right)^{2} d \nu .
$$

Since

$$
\begin{aligned}
& \left(f_{m}\left(\tau_{+}\left(v_{j}\right)\right)-f_{m}\left(\tau_{-}\left(v_{j}\right)\right)\right)^{2} \\
& \quad=\liminf _{i \rightarrow \infty}\left(f_{m}\left(\tau_{+}\left(v_{j}\right)\right)-f_{n_{i}}\left(\tau_{+}\left(v_{j}\right)\right)-f_{m}\left(\tau_{-}\left(v_{j}\right)\right)+f_{n_{i}}\left(\tau_{-}\left(v_{j}\right)\right)\right)^{2},
\end{aligned}
$$

it follows from Fatou's lemma that

$$
Q_{s}\left(f_{m}, f_{m}\right) \leq \liminf _{i \rightarrow \infty} Q_{s}\left(f_{m}-f_{n_{i}}, f_{m}-f_{n_{i}}\right)<\varepsilon
$$

Thus $\lim _{m \rightarrow \infty} Q_{s}\left(f_{m}, f_{m}\right)=0$ and $Q_{s}$ is closable.

The proof that $Q_{s}$ is Markovian is by inspection: Let $C_{-}, C_{0}, C_{+}$denote the closed subsets of $C$ for which $f \leq 0,0 \leq f \leq 1,1 \leq f$. If $\tau_{+}(v) \in C_{i}$ and $\tau_{-}(v) \in C_{j}$ then $\left|\tilde{f}\left(\tau_{+}(v)\right)-\tilde{f}\left(\tau_{-}(v)\right)\right| \leq\left|f\left(\tau_{+}(v)\right)-f\left(\tau_{-}(v)\right)\right|$ for each $i, j$. Thus $Q_{s}(\tilde{f}, \tilde{f}) \leq Q_{s}(f, f)$.

It is now possible to get a closed Dirichlet form using the following result.

Theorem 6 ([19] Thm. 2.1.1). Suppose that $Q$ is a closable Markovian symmetric form on $\mathscr{L}^{2}(X, m)$, where $X$ is a locally compact separable Hausdorff space and $m$ is a positive Radon measure on $X$ such that $\operatorname{Supp}(m)=X$. Then its smallest closed extension is a Dirichlet form.

8.2. Self-adjoint operators and operator semigroups. This section follows [19] (Ch. 1.3). Let $H$ be a real Hilbert space.

Definition 12. A family $\left\{T_{t}: t>0\right\}$ of linear operators is called a strongly continuous, symmetric, contraction semigroup if the following holds:

(i) Each $T_{t}$ is a self-adjoint contraction on $H$ (namely $\left\|T_{t}\right\| \leq 1$ ).

(ii) Semigroup property: $T_{t} T_{s}=T_{t+s}$ for $t, s>0$.

(iii) Strong continuity: $\left\|T_{t} f-f\right\| \rightarrow 0$ as $t \downarrow 0$ for all $f \in H$. 
Let $\left\{T_{t}: t>0\right\}$ be such a semigroup. Then the generator $A$ is an operator on $H$ defined by

$$
\text { Af }:=\lim _{t \downarrow 0} \frac{T_{t} f-f}{t}, \quad \operatorname{Dom}(A):=\{f \in H: A f \text { exists as a strong limit }\} .
$$

In fact, there is a one-to-one correspondence between non-positive definite self-adjoint operators on $H$ and the family of strongly continuous, symmetric, contraction semigroups. The correspondence from $A$ to $\left\{T_{t}\right\}$ is given by $T_{t}=\exp (t A)$.

Given a non-positive definite self-adjoint operator, let $Q(u, v):=\langle-A u, u\rangle$ with $\operatorname{Dom}(Q):=\operatorname{Dom}(\sqrt{-A})$. It turns out that $Q$ is a closed symmetric form on $H$. This correspondence is also one-to-one. Starting with a closed, symmetric form $Q$ on $H$ the construction of $A$ is slightly more involved. Since $Q$ is closed, $\operatorname{Dom}(Q)$ is a Hilbert space with norm $\|g\|_{1}=\|g\|_{\mathscr{L}^{2}}+Q(g, g)$. Fix $f \in H$. Then $\langle\cdot, f\rangle$ is a bounded linear functional on $\operatorname{Dom}(Q)$. Therefore, let $B f$ be the unique vector in $\operatorname{Dom}(Q)$ corresponding to this linear functional by the Riesz Representation Theorem. Let $A:=I-B^{-1}$. Then $A$ is the non-positive definite self-adjoint operator corresponding to $Q$.

Now let $H=\mathscr{L}^{2}(X, m)$ where $X$ is a locally compact separable Hausdorff space and $m$ is a positive Radon measure on $X$ such that $\operatorname{Supp}(m)=X$. A bounded linear operator $S$ on $\mathscr{L}^{2}(X, m)$ is called Markovian if $0 \leq S f \leq 1, m$-a.e. whenever $f \in$ $\mathscr{L}^{2}(X, m)$ is such that $0 \leq f \leq 1$. A strongly continuous, symmetric, contraction semigroup $\left\{T_{t}\right\}$ such that $T_{t}$ is Markovian for each $t>0$ is called a Markovian semigroup.

Theorem 7 ([19] Thm. 1.4.1). Let X be a locally compact separable Hausdorff space and $m$ a positive Radon measure on $X$ such that $\operatorname{Supp}(m)=X$. Then there is a one-to-one correspondence between Dirichlet forms on $\mathscr{L}^{2}(X, m)$ and Markovian semigroups on $\mathscr{L}^{2}(X, m)$.

8.3. The operators $\Delta_{s}$. Let $C$ be a $\zeta$-regular Cantor set with regular ultrametric $d$. Let $\mu$ be the measure constructed via the $\zeta$-function. Suppose $\mu$ is such that $\operatorname{Supp}(\mu)=C$. Then for $s \in \mathbb{R}$, the previous results give a non-positive definite self-adjoint operator $\Delta_{s}$ such that $T_{t}:=\exp \left(t \Delta_{s}\right)$ is a Markovian semigroup. The operator $\Delta_{S}$ is such that

$$
\left\langle-\Delta_{s} f, g\right\rangle=\frac{1}{2} \int_{\Upsilon(C)} \operatorname{Tr}\left(|D|^{-s}\left[D, \pi_{\tau}(f)\right]^{*}\left[D, \pi_{\tau}(g)\right]\right) d \nu(\tau)
$$

for $f, g \in \operatorname{Dom}\left(\Delta_{s}\right)$. It is important to note that $\mathcal{E} \subset \operatorname{Dom}\left(\Delta_{s}\right) \subset \operatorname{Dom}\left(\bar{Q}_{s}\right)$, where $\bar{Q}_{s}$ is the smallest closed extension of $Q_{s}$.

It is possible to calculate $\Delta_{s} \chi_{v}$ for $v \in \mathcal{V}$. Since $\chi_{v}\left(\tau_{+}(w)\right)-\chi_{v}\left(\tau_{-}(w)\right)=0$ if 
$w \nsucc v$, it follows that, for $g \in \operatorname{Dom}\left(Q_{s}\right)$,

$$
\begin{aligned}
& \left\langle-\Delta_{s} \chi_{v}, g\right\rangle \\
& =\sum_{w \succ v} \operatorname{diam}(w)^{s-2} \int_{\Upsilon(C)}\left(\chi_{v}\left(\tau_{+}(w)\right)-\chi_{v}\left(\tau_{-}(w)\right)\right)\left(g\left(\tau_{+}(w)\right)-g\left(\tau_{-}(w)\right)\right) d \nu(\tau) .
\end{aligned}
$$

Since $\tau$ is only applied to $w$, by the very definition of $v_{w}$ we may continue

$=\sum_{w \succ v} \frac{\operatorname{diam}(w)^{s-2}}{\sum_{\left(u, u^{\prime}\right) \in \mathscr{E}_{w}} \mu([u]) \mu\left(\left[u^{\prime}\right]\right)} \sum_{\left(u, u^{\prime}\right) \in \mathscr{E}_{w}} \int_{[u] \times\left[u^{\prime}\right]}\left(\chi_{v}(x)-\chi_{v}(y)\right)(g(x)-g(y)) d \mu d \mu$.

For $w$ an ancestor of $v$ let $u_{v}$ be its child that is also an ancestor of $v$. Then for any other child $u$ of $w, \chi_{u}(x)=0$ for $x \in[u]$. Thus, since $\bigcup_{\left(u_{v}, u^{\prime}\right) \in \mathscr{E}_{w}}\left[u^{\prime}\right]=[w] \cap\left[u_{v}\right]^{c}$, we have

$$
=\sum_{w \succ v} \frac{\operatorname{diam}(w)^{s-2}}{\sum_{\left(u, u^{\prime}\right) \in \mathscr{S}_{w}} \mu([u]) \mu\left(\left[u^{\prime}\right]\right)} 2 \int_{[v]} d \mu(x) \int_{[w] \cap\left[u_{v}\right]^{c}} g(x)-g(y) d \mu(y) .
$$

Consequently,

$$
\Delta_{S} \chi_{v}=-\sum_{w \succ v} \frac{\operatorname{diam}(w)^{s-2}}{\sum_{\left(u, u^{\prime}\right) \in \mathscr{E}_{w}} \mu([u]) \mu\left(\left[u^{\prime}\right]\right)} 2\left(\mu\left([w] \cap\left[u_{v}\right]^{c}\right) \chi_{v}-\mu([v]) \chi[w] \cap\left[u_{v}\right]^{c}\right)
$$

An application of this formula is given by the following:

Proposition 9. The spectrum of $\Delta_{s}$ is pure point.

Proof. Let $\mathscr{L}_{n} \subset \mathscr{L}^{2}(C, d \mu)$ be the space spanned by all $\chi_{v}$ such that $h t(v) \leq n$. Since $\mathcal{T}$ is Cantorian, it follows that $\operatorname{dim}\left(\mathscr{L}_{n}\right)<\infty$. Moreover, $\mathscr{L}_{n} \subset \mathscr{L}_{n+1}$ and $\bigcup_{n} \mathscr{L}_{n}$ is dense in $\mathscr{L}^{2}(C, d \mu)$. Equation (3) then gives that $\Delta_{s}$ leaves each $\mathscr{L}_{n}$ invariant. Since $\Delta_{s}$ restricted to each finite dimensional $\mathscr{L}_{n}$ is pure point, $\Delta_{s}$ is pure point.

\section{The triadic Cantor set}

9.1. Eigenvalues and eigenstates for $\boldsymbol{\Delta}_{\boldsymbol{s}}$ on $\boldsymbol{C}_{\mathbf{3}}$. This section will apply much of the previous machinery to the triadic Cantor set. Let $C_{3}$ denote the triadic Cantor set seen as a subset of the interval $[0,1]$. As seen in Example $1, C_{3}$ is the boundary of the infinite binary tree $\partial \mathcal{T}_{2}$ and has a natural homeomorphism with $\{0,1\}^{\mathbb{N}}$ by

$$
\phi(\omega)=\sum_{n=0}^{\infty} \frac{2 \omega_{n}}{3^{n+1}}, \quad \omega=\left\{\omega_{n}\right\}_{n \in \mathbb{N}} \in\{0,1\}^{\mathbb{N}} .
$$


Let $d$ be the regular ultrametric corresponding to the weight $\epsilon(v)=3^{- \text {ht }(v)}$. Then for $x, y \in C_{3}$,

$$
\frac{d(x, y)}{3} \leq|x-y| \leq d(x, y)
$$

and thus $d$ is metrically equivalent to the Euclidean metric. Then

$$
\zeta(s)=\sum_{n=0}^{\infty}\left(\frac{2}{3^{s}}\right)^{n}
$$

and therefore has abscissa of convergence $s_{0}=\ln 2 / \ln 3$. This pole is clearly a simple pole. For any $v \in \mathcal{V}$,

$$
\frac{1}{2} \operatorname{Tr}\left(|D|^{-s} \pi_{\tau}\left(\chi_{v}\right)\right)=\sum_{w \preceq v} \operatorname{diam}(w)^{s}=\operatorname{diam}(v)^{s} \zeta(s)
$$

since the subtree starting at $v$ is identical to the tree starting at the root. Consequently, $\mu\left(\chi_{v}\right)=\operatorname{diam}(v)^{s_{0}}$. Thus $\mu(f)$ is defined on all characteristic functions and can be extended to all continuous functions. Therefore, $C_{3}$ is $\zeta$-regular and

$$
\mu([v])=\operatorname{diam}(v)^{s_{0}}=\frac{1}{3^{s_{0} \mathrm{ht}(v)}}=\frac{1}{2^{\mathrm{ht}(v)}} .
$$

Since $\operatorname{Supp}(\mu)=C_{3}, \Delta_{s}$ can be defined on $\mathscr{L}^{2}\left(C_{3}, d \mu\right)$. Equation (3) then gives that for $v=v_{0} \ldots v_{n} \in \mathcal{V}$ with $n \geq 1$,

$$
\Delta_{s} \chi_{v}=-\sum_{j=0}^{n-1} \frac{3^{j(2-s)}}{2^{-(2 j+1)}} 2\left(2^{-(j+1)} \chi_{v}-2^{-n} \chi_{[w] \cap\left[u_{v}\right]^{c}}\right) .
$$

Letting $\bar{a}=1-a$ for $a \in\{0,1\}$, this becomes

$$
\Delta_{s} \chi_{v}=-2 \sum_{j=0}^{n-1}\left(\frac{2}{3^{s-2}}\right)^{j} \chi_{v}+\frac{4}{2^{n}} \sum_{j=0}^{n-1}\left(\frac{4}{3^{s-2}}\right)^{j} \chi_{v_{0} \ldots v_{j}} \bar{v}_{j+1} .
$$

This formula can be used to find the eigenstates of $\Delta_{S}$.

Definition 13. Let $\mathcal{W}$ be the set of infinite sequences $\omega=\omega_{1} \omega_{2} \cdots \in\{0,1\}^{N^{+}}$such that all but a finite number of $\omega_{k}$ 's are 0 . Let $|\omega|$ be the maximum integer $k$ such that $\omega_{k}=1$, with the convention that $|\omega|=0$ if $\omega=00 \ldots$ The Haar function $\phi_{\omega}$ is defined by

$$
\phi_{\omega}=\sum_{v \in\{0,1\}^{n}}(-1)^{\omega \cdot v} \chi_{v}, \quad \omega \cdot v=\sum_{k=1}^{n} \omega_{k} v_{k}
$$

for any $n \geq|\omega|$. 
Clearly $\chi_{v_{1} \ldots v_{N} 0}+\chi_{v_{1} \ldots v_{N} 1}=\chi_{v_{1} \ldots v_{N}}$. Moreover, if $N=|\omega|$ then $\omega_{N+m}=0$ for $m>0$, so

$$
\sum_{v \in\{0,1\}^{N+1}}(-1)^{\omega \cdot v} \chi_{v}=\sum_{v \in\{0,1\}^{N}}(-1)^{\omega \cdot v}\left(\chi_{v_{1} \ldots v_{N} 0}+\chi_{v_{1} \ldots v_{N} 1}\right)=\sum_{v \in\{0,1\}^{n}}(-1)^{\omega \cdot v} \chi_{v} .
$$

Therefore, $\phi_{\omega}$ does not depend on the choice of $n$ and $\phi_{\omega}$ is well defined. Moreover, it is straightforward to check that the Haar functions are orthonormal in the sense that $\left\langle\phi_{\omega}, \phi_{\sigma}\right\rangle=\delta_{\omega, \sigma}$ for $\omega, \sigma \in \mathcal{W}$. In addition,

$$
\chi_{v}=\frac{1}{2^{n}} \sum_{u \in\{0,1\}^{n}}(-1)^{v \cdot u} \phi_{u 00 \ldots}
$$

for $v \in \mathcal{V}$ and thus the Haar functions are an orthonormal basis for $\mathscr{L}^{2}\left(C_{3}, d \mu\right)$. The importance of the Haar functions comes from the following theorem.

Theorem 8. Let $C_{3}$ be the triadic Cantor set with the regular ultrametric $d$ given above. Let $\mu$ be its associated measure. Then the following is true:

(i) The eigenstates of $\Delta_{s}$ are given by the Haar functions $\phi_{\omega}$ with $\omega \in \mathcal{W}$.

(ii) The eigenvalues of $\Delta_{s}$ are given by $\lambda_{0}=0$ and for $n \geq 1$,

$$
-\lambda_{n}=-2\left(1+3^{s_{0}+2-s}+\cdots+\left(3^{s_{0}+2-s}\right)^{n-2}+2\left(3^{s_{0}+2-s}\right)^{n-1}\right) .
$$

(iii) The degeneracy of $\lambda_{n}$ is $2^{n-1}$ for $n \geq 1$ whereas $\lambda_{0}$ is simple.

(iv) For $s>s_{0}+2, \Delta_{s}$ is bounded and is a compact perturbation of a multiple of the identity.

(v) For $s \leq s_{0}+2, \Delta_{s}$ has compact resolvent.

(vi) For $s<s_{0}+2$, the density of states $\mathcal{N}(\lambda)$ given by the dimension of the spectral space corresponding to eigenvalues whose magnitude is less than or equal to $\lambda$ satisfies

$$
\mathcal{N}(\lambda) \stackrel{\lambda \uparrow \infty}{\sim} 2\left(\frac{\lambda}{2 k}\right)^{s_{0} /\left(2+s_{0}-s\right)}(1+o(1))
$$

where $k=1 /\left(1-3^{s-2-s_{0}}\right)+1$.

Remark 2. On a compact Riemannian manifold $M$, the Laplacian is an unbounded operator with compact resolvent. Moreover, Weyl's theorem says that if $m$ is the dimension of $M$, then $\mathcal{N}(\lambda) \sim c_{0} \lambda^{m / 2}$ as $\lambda \rightarrow \infty$ for an appropriate constant $c_{0}$. The constant $c_{0}$ is not arbitrary and actually gives the volume of the unit ball in the cotangent bundle over the manifold. In any case, the previous theorem shows that if $\Delta_{s}$ is interpreted as the Laplacian on a compact Riemannian manifold, then $m=2 s_{0} /\left(2+s_{0}-s\right)$ gives the Riemannian dimension of this noncommutative manifold. By analogy, this suggests that $\Delta_{s_{0}}$ is the appropriate Laplacian on $C_{3}$ since it gives Riemannian dimension $s_{0}$. 
Proof. Using Equation (4) and the definition of the Haar function, for $\omega \in \mathcal{W}$ with $|\omega|=n>0$, we obtain that

$$
\begin{aligned}
-\Delta_{s} \phi_{\omega} & =\sum_{v \in\{0,1\}^{n}}(-1)^{\omega \cdot v}\left(2 \sum_{j=0}^{n-1}\left(\frac{2}{3^{s-2}}\right)^{j} \chi_{v}-\frac{4}{2^{n}} \sum_{j=0}^{n-1}\left(\frac{4}{3^{s-2}}\right)^{j} \chi_{v_{0} \ldots v_{j} \bar{v}_{j+1}}\right) \\
& =2 \sum_{j=0}^{n-1}\left(\frac{2}{3^{s-2}}\right)^{j} \phi_{\omega}-\frac{4}{2^{n}} \sum_{j=0}^{n-1}\left(\frac{4}{3^{s-2}}\right)^{j} \sum_{v \in\{0,1\}^{n}}(-1)^{\omega \cdot v} \chi_{v_{0} \ldots v_{j}} \bar{v}_{j+1} .
\end{aligned}
$$

For $j<n-1$ the last sum on the right-hand side vanishes and for $j=n-1$ we get

$$
\sum_{v \in\{0,1\}^{n}}(-1)^{\omega \cdot v} \chi_{v_{0} \ldots v_{j}} \bar{v}_{j+1}=-\phi_{\omega}
$$

since $(-1)^{v_{n}}=-(-1)^{\bar{v}_{n}}$. Consequently,

$$
\Delta_{s} \phi_{\omega}=-\left(2 \sum_{j=0}^{n-1}\left(3^{s_{0}+2-s}\right)^{j}+2\left(3^{s_{0}+2-s}\right)^{n-1}\right) \phi_{\omega}
$$

Therefore, the Haar basis is an eigenbasis for $\Delta_{s}$ and the corresponding eigenvalues are precisely the $-\lambda_{n}$ 's given in the statement of the theorem. Since there are exactly $2^{n-1}$ sequences $\omega \in \mathcal{W}$ with $|\omega|=n$ for $n>0$, the degeneracy of $-\lambda_{n}$ is $2^{n-1}$.

If $3^{s_{0}+2-s}<1$, that is, if $s>s_{0}+2$ then, as $n \rightarrow \infty$,

$$
-\lambda_{n}=-2 \sum_{j=0}^{n-1}\left(3^{s_{0}+2-s}\right)^{j}+2\left(3^{s_{0}+2-s}\right)^{n-1} \rightarrow-\frac{2}{1-3^{s_{0}+2-s}}=:-\lambda_{\infty} .
$$

Hence, $\Delta_{s}$ is bounded and $\Delta_{s}+\lambda_{\infty} \mathbf{1}$ is compact.

If $s=s_{0}+2$, then $3^{s_{0}+2-s}=1$ and $-\lambda_{n}=-2(n+1)$. Therefore, $\left(\Delta_{s}^{2}+1\right)^{-1}$ is compact and $\Delta_{s}$ has compact resolvent. If $s<s_{0}+2$, then $3^{s_{0}+2-s}>1$ and

$$
-\lambda_{n}=-2\left(3^{s_{0}+2-s}\right)^{n-1}\left(\frac{1-\left(3^{s-2-s_{0}}\right)^{n}}{1-3^{s-2-s_{0}}}+1\right) .
$$

Therefore, $\left(\Delta_{s}^{2}+1\right)^{-1}$ is compact and $\Delta_{s}$ has compact resolvent. Moreover, if $N(\lambda)$ is such that

$$
\lambda=2\left(3^{s_{0}+2-s}\right)^{N(\lambda)-1}\left(\frac{1-\left(3^{s-2-s_{0}}\right)^{N(\lambda)}}{1-3^{s-2-s_{0}}}+1\right),
$$

then if $k:=1 /\left(1-3^{s-2-s_{0}}\right)+1$, it follows that

$$
N(\lambda)=1+\frac{\ln \left(\lambda+2\left(3^{s_{0}+2-s}-1\right)^{-1}\right)-\ln 2 k}{\ln 2-(s-2) \ln 3} .
$$


Now

$$
\lim _{\lambda \rightarrow \infty}\left(N(\lambda)-\frac{\ln (\lambda /(2 k))}{\ln 2-(s-2) \ln 3}\right)=0
$$

Thus, since

$$
\mathcal{N}(\lambda)=1+\sum_{n \geq 1, \lambda_{n} \leq \lambda} 2^{n-1}=2^{N(\lambda)}
$$

we obtain that

$$
\mathcal{N}(\lambda) \sim 2\left(\frac{\lambda}{2 k}\right)^{s_{0} /\left(2+s_{0}-s\right)}(1+o(1))
$$

as $\lambda \rightarrow \infty$, as desired.

9.2. Diffusion on $\boldsymbol{C}_{\mathbf{3}}$. Having computed the eigenstates and eigenvalues of $\Delta_{S}$, it is now possible to get an explicit description of its associated Markovian semigroup $\left\{\exp \left(t \Delta_{s}\right)\right\}_{t>0}$. In order to do so, let

$$
\kappa_{n}(x, y):= \begin{cases}1 & \text { if } d(x, y)=3^{-n} \\ 0 & \text { otherwise. }\end{cases}
$$

Theorem 9. Under the assumptions of Theorem 8 and for $s<s_{0}+2$, the following holds:

(i) Let the heat kernel $K_{t}(x, y)$ be defined by

$$
\left\langle f, e^{t \Delta_{s}} g\right\rangle=\int_{C_{3} \times C_{3}} f(x) K_{t}(x, y) g(y) d \mu(x) d \mu(y)
$$

for $f, g \in \mathscr{L}^{2}\left(C_{3}, d \mu\right)$. Then $K_{t}(x, y)=\sum_{n=0}^{\infty} \kappa_{n}(x, y) a_{n}(t, s)$ where

$$
a_{n}(t, s)=1-2^{n} e^{-t \lambda_{n+1}}+\sum_{m=1}^{n} 2^{m-1} e^{-t \lambda_{m}}
$$

for $n \geq 1$ and $a_{0}=1-e^{-t \lambda_{1}}$. Moreover, $K_{t}(x, y)$ is positive for all $x, y \in C_{3}$ and $t>0$. In addition, $K_{t} \in \mathscr{L}^{\infty}\left(C_{3} \times C_{3}, \mu \times \mu\right)$ for $t>0$.

(ii) The Markovian semigroup $\left\{e^{t \Delta_{s}}\right\}$ defines a Markov process $\left(X_{t}\right)_{t \geq 0}$ with values in $C_{3}$ defined by

$$
\mathbb{E}\left(f_{1}\left(X_{t_{1}}\right) \ldots f_{n}\left(X_{t_{n}}\right)\right)=\left\langle 1, \hat{f}_{n} e^{\left(t_{n}-t_{n-1}\right) \Delta_{s}} \ldots \hat{f}_{1} e^{t_{1} \Delta_{s}} 1\right\rangle,
$$

where $f_{k} \in \mathcal{C}(C), \hat{f}$ denotes the operator on $\mathscr{L}^{2}\left(C_{3}, d \mu\right)$ given by multiplication by $f$, and where $t_{n}>\cdots>t_{1}>0$. This Markov process is stationary and satisfies the following for $s$ fixed:

$$
\begin{aligned}
\mathbb{E}\left(d\left(X_{t_{0}}, X_{t_{0}+t}\right)^{\beta}\right) & \\
& \stackrel{t \downarrow 0}{\sim}\left(\frac{\lambda_{1}}{2}+\frac{1}{2} \sum_{n=1}^{\infty}\left(\frac{1}{2 \cdot 3^{\beta}}\right)^{n}\left(2^{n} \lambda_{n+1}-\sum_{m=1}^{n} 2^{m-1} \lambda_{m}\right)\right) t(1+o(1))
\end{aligned}
$$




$$
\begin{aligned}
& \text { for } \beta>s_{0}+2-s \text { and } \\
& \mathbb{E}\left(d\left(X_{t_{0}}, X_{t_{0}+t}\right)^{\beta}\right) \stackrel{t \downarrow 0}{\sim} \frac{1}{2 \beta \ln 3}\left(\frac{1}{1-3^{-\beta}}+1\right)\left(1-\frac{1}{3^{\beta+s_{0}-1}}\right) t \ln (1 / t)(1+o(1)) \\
& \text { for } \beta=s_{0}+2-s \text {. For } \beta<s_{0}+2-s \text {, we have } \\
& \mathbb{E}\left(d\left(X_{t_{0}}, X_{t_{0}+t}\right)^{\beta}\right)=O\left(t^{\beta /\left(s_{0}+2-s\right)} \ln (1 / t)\right) .
\end{aligned}
$$

Remark 3. The previous section had suggested that $\Delta_{S_{0}}$ is the proper generalization of the Laplacian to the Cantor set. Classical Brownian motion on the real line is generated by the Laplacian and satisfies $\mathbb{E}\left(\left|X_{t_{0}}-X_{t+t_{0}}\right|^{2}\right)=|t|$. For $s=s_{0}$, $\mathbb{E}\left(d\left(X_{t_{0}}, X_{t_{0}+t}\right)^{2}\right) \sim t \ln (1 / t)$ and so there is a subdominant contribution by a term of order $\ln (1 / t)$. For $\beta=2$ this subdominant contribution only appears for $s \leq s_{0}$ and therefore suggests that on the Cantor set something special is happening at $s=s_{0}$ as the subdominant term $t \ln (1 / t)$ takes over from the term $t$, which dominates for $s>s_{0}$. A further understanding of this phenomenon needs to be investigated, although presumably this logarithmic singularity comes from the fact that $X_{t}$ describes a jump process across the gaps of the Cantor set.

Proof. Because of the spectral decomposition of $\Delta_{s}$ given in Theorem 8, we have

$$
e^{t \Delta_{s}}=\sum_{n=0}^{\infty} e^{-t \lambda_{n}} \Pi_{n}
$$

where $\Delta_{s}$ is the spectral projection onto the eigenspace of $\Delta_{s}$ corresponding to the eigenvalue $-\lambda_{n}$. For $n=0, \Pi_{0}=\left|\phi_{00} \ldots\right\rangle\left\langle\phi_{00} \ldots|=| 1\right\rangle\langle 1|$. For $n \geq 1$,

$$
\Pi_{n}=\sum_{\sigma_{1}, \ldots, \sigma_{n-1} \in\{0,1\}}\left|\phi_{\sigma_{1} \ldots \sigma_{n-1} 100 \ldots}\right\rangle\left\langle\phi_{\sigma_{1} \ldots \sigma_{n-1} 100 \ldots}\right|
$$

since $\phi_{\sigma_{1} \ldots \sigma_{n-1} 100 \ldots}$ generate the eigenspace of Haar functions $\phi_{\omega}$ with $|\omega|=n$. By the definition of the Haar function,

$$
\begin{aligned}
\Pi_{n} & =\sum_{\substack{u_{k}, v_{k} \in\{0,1\} \\
k=1, \ldots, n}}(-1)^{u_{n}-v_{n}}\left|\chi_{u}\right\rangle\left\langle\chi_{v}\right| \sum_{\sigma_{1}, \ldots, \sigma_{n-1} \in\{0,1\}} \prod_{k=1}^{n-1}(-1)^{\left(u_{k}-v_{k}\right) \sigma_{k}} \\
& =2^{n-1} \sum_{u \in\{0,1\}^{n-1}}\left|\chi_{u 0}\right\rangle\left\langle\chi_{u 0}|-| \chi_{u 0}\right\rangle\left\langle\chi_{u 1}|-| \chi_{u 1}\right\rangle\left\langle\chi_{u 0}|+| \chi_{u 1}\right\rangle\left\langle\chi_{u 1}\right| .
\end{aligned}
$$

Now $\left|\chi_{u}\right\rangle\left\langle\chi_{v}\right|$ is the operator with functional kernel $\chi_{u}(x) \chi_{v}(y)$. Because

$$
\sum_{u \in\{0,1\}^{n-1}} \chi_{u 0}(x) \chi_{u 0}(y)+\chi_{u 1}(x) \chi_{u 1}(y)= \begin{cases}1 & \text { if } d(x, y) \leq 3^{-n} \\ 0 & \text { otherwise }\end{cases}
$$


and

$$
\sum_{u \in\{0,1\}^{n-1}} \chi_{u 0}(x) \chi_{u 1}(y)+\chi_{u 1}(x) \chi_{u 0}(y)= \begin{cases}1 & \text { if } d(x, y)=3^{-n+1} \\ 0 & \text { otherwise }\end{cases}
$$

it follows that

$$
\Pi_{n}(x, y)= \begin{cases}2^{n-1} & \text { if } d(x, y) \leq 3^{-n}, \\ -2^{n-1} & \text { if } d(x, y)=3^{-n+1}, \\ 0 & \text { otherwise, }\end{cases}
$$

where $\Pi_{n}(x, y)$ is the functional kernel of the operator $\Pi_{n}$. Using the functions $\kappa_{n}$, this becomes

$$
\Pi_{n}(x, y)=2^{n-1}\left(-\kappa_{n-1}(x, y)+\sum_{m \geq n} \kappa_{m}(x, y)\right)
$$

Therefore,

$$
\begin{aligned}
K_{t}(x, y) & =\sum_{n=0}^{\infty} e^{-t \lambda_{n}} \Pi_{n}(x, y) \\
& =\kappa_{0}(x, y)\left(1-e^{-t \lambda_{1}}\right)+\sum_{n=1}^{\infty} \kappa_{n}(x, y)\left(1-2^{n} e^{-t \lambda_{n+1}}+\sum_{m=1}^{n} 2^{m-1} e^{-t \lambda_{m}}\right) .
\end{aligned}
$$

The convergence of $K_{t}(x, y)$ in $\mathscr{L}^{2}\left(C_{3} \times C_{3}, \mu \times \mu\right)$ is shown as follows. To begin,

$$
\kappa_{n}(x, y)=\sum_{v \in\{0,1\}^{n}} \chi_{v 0}(x) \chi_{v 1}(y)+\chi_{v 1}(x) \chi_{v 0}(y)
$$

gives that

$$
\int_{C_{3} \times C_{3}} \kappa_{n}(x, y)^{2} d \mu(x) d \mu(y)=\sum_{v \in\{0,1\}^{n}} \frac{2}{2^{2 n+2}}=\frac{1}{2^{n+1}} .
$$

Therefore, the corresponding norm in $\mathscr{L}^{2}\left(C_{3} \times C_{3}, \mu \times \mu\right)$ is $\left\|\kappa_{n}\right\|_{2}=2^{-(n+1) / 2}$. The coefficients of the $\kappa_{n}$ 's in $K_{t}$ are positive for $t>0$ since

$$
a_{n}(t, s)=2^{n}\left(1-e^{-t \lambda_{n+1}}\right)-\sum_{m=1}^{n} 2^{m-1}\left(1-e^{-t \lambda m}\right)>1-e^{-t \lambda_{n+1}}>0 .
$$

It is straightforward to show that for $t>0$,

$$
1+\sum_{m=1}^{\infty} 2^{m-1} e^{-t \lambda_{m}}=M_{S}(t)<\infty
$$


for all $s<s_{0}+2$ and thus $0<a_{n}(t, s)<M_{s}(t)$. Since the $\kappa_{n}$ 's have disjoint support, $K_{t}$ is bounded and $K_{t} \in \mathscr{L}^{\infty}\left(C_{3} \times C_{3}, \mu \times \mu\right)$.

The definition of the stochastic process $\left(X_{t}\right)_{t \geq 0}$ is standard and results from the Chapman-Kolmogorov equations. It gives a way to evaluate $\mathbb{E}\left(d\left(X_{t_{0}}, X_{t_{0}+t}\right)^{\beta}\right)$ by

$$
\mathbb{E}\left(d\left(X_{t_{0}}, X_{t_{0}+t}\right)^{\beta}\right)=\int_{C_{3} \times C_{3}} K_{t}(x, y) d(x, y)^{\beta} d \mu(x) d \mu(y) .
$$

Thus

$$
\mathbb{E}\left(d\left(X_{t_{0}}, X_{t_{0}+t}\right)^{\beta}\right)=\frac{1}{2} \sum_{n=0}^{\infty}\left(\frac{1}{2 \cdot 3^{\beta}}\right)^{n} a_{n}(t, s) .
$$

Now for $t>0$ and $\beta>s_{0}+2-s$,

$$
\frac{1}{t} \mathbb{E}\left(d\left(X_{t_{0}}, X_{t_{0}+t}\right)^{\beta}\right) \leq \frac{1}{2 t} \sum_{n=0}^{\infty} \frac{1}{3^{\beta n}}\left(1-e^{-t \lambda_{n+1}}\right) \leq \frac{1}{2} \sum_{n=0}^{\infty} \frac{1}{3^{\beta n}} \lambda_{n+1}<\infty
$$

and therefore by dominated convergence,

$$
\lim _{t \rightarrow 0} \frac{\mathbb{E}\left(d\left(X_{t_{0}}, X_{t_{0}+t}\right)^{\beta}\right)}{t}=\frac{\lambda_{1}}{2}+\frac{1}{2} \sum_{n=1}^{\infty}\left(\frac{1}{2 \cdot 3^{\beta}}\right)^{n}\left(2^{n} \lambda_{n+1}-\sum_{m=1}^{n} 2^{m-1} \lambda_{m}\right),
$$

and this limit is positive and finite. For $\beta=s_{0}+2-s$, let $N_{t}=\ln (1 / t) /(\beta \ln 3)$. First of all,

$$
\frac{1}{2} \sum_{n=N_{t}+1}^{\infty}\left(\frac{1}{2 \cdot 3^{\beta}}\right)^{n} a_{n}(t, s)<\frac{1}{2} \sum_{n=N_{t}+1}^{\infty}\left(\frac{1}{3^{\beta}}\right)^{n}=\frac{t}{2 \cdot 3^{\beta}} \frac{1}{1-3^{-\beta}}
$$

and

$$
\begin{aligned}
\frac{1}{2} \sum_{n=N_{t}+1}^{\infty}\left(\frac{1}{2 \cdot 3^{\beta}}\right)^{n} a_{n}(t, s) & >\frac{1}{2}\left(1-e^{-t \lambda_{N_{t}+2}}\right) \sum_{n=N_{t}+1}^{\infty}\left(\frac{1}{2 \cdot 3^{\beta}}\right)^{n} \\
& >\left(1-e^{-t \lambda_{1}}\right) \frac{t^{1+s_{0} / \beta}}{4 \cdot 3^{\beta}} \frac{1}{1-2^{-1} 3^{-\beta}}
\end{aligned}
$$

By taking a Taylor expansion,

$$
2^{n} t \lambda_{n+1}-2^{n} \frac{t^{2} \lambda_{n+1}^{2}}{2}-t \sum_{m=1}^{n} 2^{m-1} \lambda_{m} \leq a_{n}(t, s)
$$

and

$$
a_{n}(t, s) \leq 2^{n} t \lambda_{n+1}-t \sum_{m=1}^{n} 2^{m-1} \lambda_{m}-\frac{t^{2}}{2} \sum_{m=1}^{n} 2^{m-1} \lambda_{m}^{2}
$$


Now

$$
\frac{1}{2} \sum_{n=1}^{N_{t}}\left(\frac{1}{2 \cdot 3^{\beta}}\right)^{n} 2^{n} t^{2} \lambda_{n+1}^{2}<t^{2}\left(\frac{1}{1-3^{-\beta}}+1\right) \sum_{n=1}^{N_{t}} 3^{n \beta}<c_{0} t^{2} 3^{\beta N_{t}}=c_{0} t
$$

for some constant $c_{0}>0$. Similarly, there exists $c_{1}>0$ such that

$$
\sum_{n=1}^{N_{t}}\left(\frac{1}{2 \cdot 3^{\beta}}\right)^{n} \frac{t^{2}}{2} \sum_{m=1}^{n} 2^{m-1} \lambda_{m}^{2}<c_{1} t
$$

Since

$$
\begin{aligned}
& \left(\frac{1}{2 \cdot 3^{\beta}}\right)^{n}\left(2^{n} \lambda_{n+1}-\sum_{m=1}^{n} 2^{m-1} \lambda_{m}\right) \\
& \quad=\left(\frac{1}{1-3^{-\beta}}+1\right)\left(1-\sum_{m=1}^{n}\left(2 \cdot 3^{\beta}\right)^{-m}\right)-\frac{3^{-\beta}}{1-3^{-\beta}}\left(\frac{1}{2 \cdot 3^{\beta}}\right)^{n}
\end{aligned}
$$

it follows that

$$
\begin{aligned}
& \frac{t}{2} \sum_{n=1}^{N_{t}}\left(\frac{1}{2 \cdot 3^{\beta}}\right)^{n}\left(2^{n} \lambda_{n+1}-\sum_{m=1}^{n} 2^{m-1} \lambda_{m}\right) \\
& \quad=\frac{1}{2}\left(\frac{1}{1-3^{-\beta}}+1\right)\left(1-\frac{1}{3^{\beta+s_{0}}-1}\right) t N_{t}+c_{2} t\left(1-t^{1+s_{0} / \beta}\right),
\end{aligned}
$$

where $c_{2}>0$ is a constant. Consequently,

$$
\mathbb{E}\left(d\left(X_{t_{0}}, X_{t_{0}+t}\right)^{\beta}\right) \stackrel{t \downarrow 0}{\sim} \frac{1}{2 \beta \ln 3}\left(\frac{1}{1-3^{-\beta}}+1\right)\left(1-\frac{1}{3^{\beta+s_{0}-1}}\right) t \ln (1 / t)(1+o(1))
$$

for $\beta=s_{0}+2-s$. The proof that $\mathbb{E}\left(d\left(X_{t_{0}}, X_{t_{0}+t}\right)^{\beta}\right)=O\left(t^{\beta /\left(s_{0}+2-s\right)} \ln (1 / t)\right)$ is the same as above using the fact that $1-e^{-x} \leq x^{\alpha}$ for $0 \leq \alpha \leq 1$.

9.3. Relationship with the Vladimirov operator. Let $p$ be a prime number. It is a basic fact (see [32]) that the Cantor set is homeomorphic to the $p$-adic integers, $\mathbb{Z}_{p}$. In fact, $\mathbb{Z}_{p}$ is the boundary of the tree $\mathcal{T}_{p}$ where every vertex has exactly $p$ children and the weight function is $\epsilon(v)=p^{-\operatorname{ht}(v)}$ for $v \in \mathcal{V}$. The $p$-adic numbers are the completion of $\mathbb{Q}$ with respect to this ultrametric $|\cdot|_{p}$ and $\mathbb{Z}_{p}$ is then the closed unit disc in $\mathbb{Q}_{p}$. The Vladimirov operator [33] is constructed using the field structure of $\mathbb{Q}_{p}$. It is defined by

$$
(\mathscr{D} \psi)(x)=\frac{p^{2}}{p+1} \int_{\mathbb{Q}_{p}} \frac{\psi(x)-\psi(y)}{|x-y|_{p}^{2}} d y,
$$

where $\psi: \mathbb{Q}_{p} \rightarrow \mathbb{R}$ is a locally constant function with compact support and the measure $d y$ is the Haar measure on $\mathbb{Q}_{p}$. 
Proposition 10. For $z=v_{0} v_{1} \cdots \in \partial \mathcal{T}_{2}$ and $f \in \mathcal{E}$,

$$
(\mathscr{D} f)(z)=\frac{1}{3} \lim _{n \rightarrow \infty} \frac{1}{\mu\left(\left[v_{n}\right]\right)}\left\langle\chi_{v_{n}},-\Delta_{2} f\right\rangle .
$$

Proof. From Section 8.3 it follows that

$$
\left\langle\chi_{v_{n}},-\Delta_{2} f\right\rangle=\int_{\left[v_{n}\right]} d \mu(x) \sum_{j=0}^{n-1} \frac{4}{\mu\left(\left[v_{0} \ldots v_{j-1}\right]\right)^{2}} \int_{\left[v_{0} \ldots v_{j-1} \bar{v}_{j}\right]} f(x)-f(y) d \mu(y) .
$$

But for $x \in\left[v_{0} \ldots v_{j}\right]$ and $y \in\left[v_{0} \ldots v_{j-1} \bar{v}_{j}\right]$, we have $|x-y|_{2}=\mu\left(\left[v_{0} \ldots v_{j-1}\right]\right)$. Therefore,

$$
\left\langle\chi v_{n},-\Delta_{2} f\right\rangle=4 \int_{\left[v_{n}\right]} d \mu(x) \int_{\left[v_{n}\right]^{c}} \frac{f(x)-f(y)}{|x-y|_{2}^{2}} d \mu(y)
$$

and the result follows.

Because $|D|^{-1} D=F$ is the phase of the operator $D$, this result shows that the Vladimirov operator does not take the metric on $C_{3}$ into account. This makes sense because the Vladimirov operator was created using the 2-adic metric which comes from the measure and not from the metric on $C_{3}$.

\section{Conclusion and open problems}

In the present article the appropriate machinery has been constructed from Noncommutative Geometry to investigate various examples of ultrametric Cantor sets as Noncommutative Riemannian spaces. The study of such examples will be covered in a subsequent paper by the authors. Many of the results on the triadic Cantor set hold for a much larger class of examples. In particular, it can be shown that every attractor of a self-similar iterated function system that satisfies the strong separability condition is such that its natural metric coming from the attractor is equivalent to a regular ultrametric. This result also holds for cookie-cutter systems, which is a class of Cantor sets that includes many Julia sets. Basic definitions of these two classes of Cantor sets can be found in [16]. An important generalization by the authors of some of the results for the triadic Cantor set is the following.

Theorem 10. Let $C$ be the attractor of a self-similar iterated function system that satisfies the strong separability condition. Then the following is true:

(i) $C$ is a $\zeta$-regular Cantor set with respect to a regular ultrametric that is metrically equivalent to the natural metric coming from the iterated function system. 
(ii) Up to a constant, $\mu$ is equal to the $s_{0}$-Hausdorffmeasure where $s_{0}$ is the similarity dimension of $C$.

It is unclear whether $\zeta$-regularity is enough of a constraint in general to guarantee that the Hausdorff dimension and upper box dimension coincide. Moreover, it is an open problem to find conditions under which the measure $\mu$ of a $\zeta$-regular Cantor set is actually the Hausdorff measure.

Another important class of examples is given by the transversal $\Xi$ of an aperiodic, repetitive Delone set of finite type [4]. Such an example can be given a natural tree structure coming from its patches. The Voronoi metric is then a natural regular ultrametric on $\Xi$. The special case of the Fibonacci tiling has been investigated by the authors. It can be shown that it is a $\zeta$-regular Cantor set with $\zeta$-function equal to the Riemann $\zeta$-function plus a small perturbation. Because the Riemann $\zeta$-function has an isolated pole at $z=1$, the Fibonacci tiling has upper box dimension equal to 1 . The algorithmic complexity of the Fibonacci tiling is also 1 and it seems that the upper box dimension and algorithmic complexity should agree for more general tilings. In [28], Lapidus proposes a new definition of fractality as a set whose $\zeta$-function has non-real singularities in the positive half-plane. The Fibonacci tiling then provides a counterexample to this definition since it has only a singularity at $z=1$ in the positive half-plane. The Fibonacci tiling also has a natural construction as a cut-and-project tiling. The transversal space of the cut-and-project tiling gives a natural embedding (but not an isometry) of the transversal of the Fibonacci tiling into $\mathbb{R}$. The authors have shown that the measure $\mu$ associated to the $\zeta$-function of $\Xi$ is then the Lebesgue measure coming from this embedding. For this reason one can argue whether the transversal of the Fibonacci tiling is really a fractal. A generalization of this fact to the transversal of a cut-and-project tiling is a subject of future research.

\section{A. Proof of Michon's correspondence}

A.1. Proof of Proposition 5. Given a regular ultrametric $d$, the equivalence relation $\stackrel{\varepsilon}{\sim}$ given by $\varepsilon$-chains are shown to be a profinite structure.

(i) For $y \in[x]_{\varepsilon}, B_{\varepsilon}(y):=\{z \in C: d(z, y)<\varepsilon\} \subset[x]_{\varepsilon}$. Thus $[x]_{\varepsilon}$ is open. Therefore $R_{\varepsilon}=\bigcup_{x \in C}[x]_{\varepsilon} \times[x]_{\varepsilon}$ is open. A compact metric space is totally bounded, so there exists $\varepsilon$ such that $R_{\varepsilon}=C \times C$.

(ii) Let $x \stackrel{\mathcal{E}}{\sim} y$. Then there exists $x_{0}=x, x_{1}, \ldots, x_{n}=y$ with $d\left(x_{i}, x_{i+1}\right)<\varepsilon$. If $\eta=\left(\max \left\{d\left(x_{i}, x_{i+1}\right): 0 \leq i<n\right\}\right) / 2$, then $x \stackrel{\eta}{\sim} y$ with $\eta<\varepsilon$.

(iii) Suppose that $[x]_{0}:=\bigcap_{\varepsilon \in \mathbb{R}}+[x]_{\varepsilon}$ is the disjoint union of two closed sets $U$ and $V$. Since $C$ is compact, if both $U$ and $V$ are nonempty, there exists $u \in U$ and $v \in V$ such that $\operatorname{dist}(U, V)=d(u, v)>0$. But if $\eta=d(u, v) / 2$, then $u \stackrel{\eta}{\sim} v$. So $[x]_{0}$ must be connected. Thus since $C$ is totally disconnected, $[x]_{0}=\{x\}$. Therefore, $\bigcap_{\varepsilon \in \mathbb{R}}+R_{\varepsilon}=\Delta$. 
(iv) Finally, given another regular ultrametric $d^{\prime} \neq d$ then there exists $x, y \in C$ with $d(x, y) \neq d^{\prime}(x, y)$. Suppose that $d(x, y)=\varepsilon>d^{\prime}(x, y)=\varepsilon^{\prime}$. If $\eta=$ $\left(\varepsilon+\varepsilon^{\prime}\right) / 2$, then $x \stackrel{\eta}{\sim} d^{\prime} y$ but $x \stackrel{\eta}{\sim}_{d} y$, and therefore they give different profinite structures.

Conversely, given a profinite structure $\left\{R_{\varepsilon}: \varepsilon \in \mathbb{R}^{+}\right\}$on $C$ let $d(x, y):=$ $\inf \{\varepsilon: x \stackrel{\mathcal{E}}{\sim} y\}$. That $d(x, y)=0$ if and only if $x=y$ follows from the fact that $\bigcap_{\varepsilon \in \mathbb{R}^{+}} R_{\varepsilon}=\Delta$. For $x, y, z \in C$, if $x \stackrel{\varepsilon_{1}}{\sim} y$ and $y \stackrel{\varepsilon_{2}}{\sim} z$ and if $\varepsilon=\max \left\{\varepsilon_{1}, \varepsilon_{2}\right\}$, then $x \stackrel{\mathcal{E}}{\sim} z$. Thus $d(x, z) \leq \max \{d(x, y), d(y, z)\}$ and $d$ is an ultrametric. In order to show that $d$ is regular, let id: $C \rightarrow C$ be the identity map from $C$ with the original topology to $C$ with the metric topology. First of all, if $x \stackrel{a}{\sim} y$ then by (ii) $x \stackrel{a-\delta}{\sim} y$ for some $\delta>0$ and $d(x, y)<a$. Thus, $d(x, y)<\varepsilon$ if and only if $x \stackrel{\varepsilon}{\sim} y$. This gives that $B_{a}(x)=[x]_{a}$. In fact, $[x]_{\varepsilon}$ is open in the original topology. This can be seen as follows. Let $(x, y) \in C \times C$. Since $R_{\varepsilon}$ is open, there exists an open set $V \subset C \times C$ such that $(x, y) \in V \subset R_{\varepsilon}$. But $C \times C$ has the product topology and therefore there exists open sets $U_{x}, U_{y} \subset C$ such that $(x, y) \in U_{x} \times U_{y} \subset V$. For any $y \in U_{y},(x, y) \in R_{\varepsilon}$ and consequently $U_{y} \subset[x]_{\varepsilon}$ and $[x]_{\varepsilon}$ is open. Therefore, id is a continuous, bijective map from a compact space to a Hausdorff space and hence a homeomorphism. Thus, $d$ is regular.

Given two different profinite structures $\left\{R_{\varepsilon}\right\}$ and $\left\{R_{\varepsilon}^{\prime}\right\}$, then without loss of generality there exist $\varepsilon>0$ and $(x, y) \in R_{\varepsilon}$ such that $(x, y) \notin R_{\varepsilon}^{\prime}$. Suppose that $\left\{R_{\varepsilon}\right\}$ gives ultrametric $d$ and $\left\{R_{\varepsilon}^{\prime}\right\}$ gives an ultrametric $d^{\prime}$. Then by (ii), $(x, y) \in R_{\varepsilon-\delta}$ for some $\delta>0$ and $d(x, y)<\varepsilon \leq d^{\prime}(x, y)$. Consequently, $d \neq d^{\prime}$.

A.2. Proof of Proposition 6. Let $d$ be a regular ultrametric on $C$ and let $\left\{R_{\varepsilon}\right\}$ be the profinite structure corresponding to $d$. The tree $\mathcal{T}$ is built as follows. Let $\varepsilon_{0}=\inf \left\{\varepsilon: R_{\varepsilon}=C \times C\right\}$. Then $R_{\varepsilon_{0}} \neq C \times C$ since $R_{\varepsilon_{0}}=\bigcup_{\varepsilon^{\prime}<\varepsilon_{0}} R_{\varepsilon}$. Similarly, let $\varepsilon_{i+1}=\inf \left\{\varepsilon: R_{\varepsilon}=R_{\varepsilon_{i}}\right\}$. Then $\left\{\varepsilon_{i}\right\}_{i=0}^{\infty}$ is such that $R_{\varepsilon_{i}} \neq R_{\varepsilon_{i+1}}$. Let the root of $\mathcal{T}$ correspond to $C$ and let the vertices of height $n$ correspond to the equivalence classes of $R_{\varepsilon_{n-1}}$. Let the edges be defined by $[x]_{\varepsilon_{j}} \succeq[y]_{\varepsilon_{k}}$ if and only if $[x]_{\varepsilon_{j}} \supset[y]_{\varepsilon_{k}}$. Then $\mathcal{T}$ is a rooted tree with no dangling vertex. As seen in the proof of the previous proposition, every equivalence class is clopen. Thus each vertex has a finite number of children and has a descendant with more than one children. So, $\mathcal{T}$ is a Cantorian tree. In general, $\mathcal{T}$ is not reduced. However, since each vertex has a descendant with more than one child, edge reduction can be applied to each vertex with only one child without altering $\partial \mathcal{T}$. This will give a reduced tree $\mathcal{T}^{\prime}$ with vertices $\mathcal{V}^{\prime} \subset \mathcal{V}$ such that $\partial \mathcal{T}^{\prime}=\partial \mathcal{T}$ as topological spaces.

Let $\Phi: \partial \mathcal{T}^{\prime} \rightarrow C$ be defined by $\Phi\left(v_{0} v_{1} \ldots\right)=\bigcap_{i=1}^{\infty}\left[x_{i}\right]_{\varepsilon_{i}}$ where $v_{i}=\left[x_{i}\right]_{\varepsilon_{i}}$. This map is bijective and $\Phi^{-1}\left([x]_{\varepsilon_{i}}\right)=[v]$ where $v=[x]_{\varepsilon_{i}}$. Thus $\Phi$ is continuous and since $\partial \mathcal{T}^{\prime}$ is compact, $\Phi$ is a homeomorphism. By abuse of notation, let $[v]=[x]_{\varepsilon_{i}}$ if $v=[x]_{\varepsilon_{i}}$.

If $v=[x]_{\varepsilon_{k}}$ let $\epsilon(v):=\varepsilon_{k+1}$. Since $\varepsilon_{k}>0$ for all $k$, we have $\epsilon: \mathcal{V}^{\prime} \rightarrow \mathbb{R}^{+}$. 
The condition (i) in Definition 8 follows immediately. For (ii), since $\epsilon\left([x]_{\varepsilon_{k}}\right) \leq \varepsilon_{k}$ and $\varepsilon_{k} \rightarrow 0$, it follows that $\lim _{k \uparrow \infty} \epsilon\left([x]_{\varepsilon_{k}}\right) \leq \lim _{k \rightarrow \infty} \varepsilon_{k}=0$. So $\mathcal{T}^{\prime}$ is a reduced, weighted, rooted Cantorian tree.

Let $\mathcal{T}$ be a reduced, rooted Cantorian tree with weight function $\epsilon$. For $x, y \in$ $\partial \mathcal{T}=: C$, let $d(x, y)=\epsilon(x \wedge y)$ for $x \neq y$ and $d(x, x)=0$. It is straightforward to show that $d$ is an ultrametric on $C$. Given $r>0$ and $x \in C$, let $B_{r}(x):=\{y \in C$ : $d(x, y)<r\}$. By (ii), $B_{r}(x)$ has more than one point, so let $v=1$.c.p. $\left(B_{r}(x)\right)$. By the definition of $v$, for $y \in[v]$ there exists $z \in B_{r}(x)$ such that $x \wedge y \preceq x \wedge z$. Thus $d(x, y) \leq d(x, z)<r$ and therefore $[v]=B_{r}(x)$. Consequently, $B_{r}(x)$ is open in $\partial \mathcal{T}$ and $d$ is regular.

For $x, y \in[v]$ then $x \wedge y \preceq v$ and $d(x, y)=\epsilon(x \wedge y) \leq \epsilon(v)$. Thus, $\operatorname{diam}([v]) \leq$ $\epsilon(v)$. Conversely, since $v$ has more than one child, there exists $x, y \in[v]$ such that $v=x \wedge y$. Therefore, $\epsilon(v)=d(x, y) \leq \operatorname{diam}(v)$ and $\epsilon(v)=\operatorname{diam}([v])$.

Starting with a regular ultrametric $d$ on $C$, let $d_{\varepsilon}$ be the regular ultrametric obtained from the Cantorian tree $\mathcal{T}$ corresponding to $d$. Let $x, y \in C$. Then $d_{\varepsilon}(x, y)=$

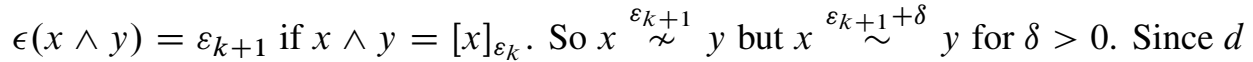
is an ultrametric, $d(x, y)=\varepsilon_{k+1}$. Thus $d=d_{\varepsilon}$ and $\partial \mathcal{T}$ is isometric to $C$.

Starting with a reduced, weighted, rooted Cantorian tree $\mathcal{T}$, let $\mathcal{T}_{d}$ be the tree obtained from the regular ultrametric $d$ corresponding to $\mathcal{T}$. Let $\Phi$ be the homeomorphism from $\partial \mathcal{T} \rightarrow \partial \mathcal{T}_{d}$. Let $\Psi: \mathcal{V} \rightarrow \mathcal{V}_{d}$ be defined by $\Psi(v)=$ 1.c.p $(\Phi([v]))$. Because each tree is reduced there is a one-to-one correspondence between clopen sets in the boundary and vertices, thus $\Psi$ is a bijection. Therefore the correspondence between reduced, weighted, rooted Cantorian trees and regular ultrametrics is indeed a bijection.

\section{References}

[1] S. Albeverio and W. Karwowski, A random walk on $p$-adics - the generator and its spectrum. Stochastic Process. Appl. 53 (1994), 1-22. Zbl 0810.60065 MR 1290704

[2] J. Bellissard, The noncommutative geometry of aperiodic solids. In Geometric and topological methods for quantum field theory (Villa de Leyva, 2001), World Sci. Publ., Singapore 2003, 86-156. Zbl 1055.81034 MR 2009996

[3] J. Bellissard, R. Benedetti, and J.-M. Gambaudo, Spaces of tilings, finite telescopic approximations and gap-labeling. Comm. Math. Phys. 261 (2006), 1-41. Zbl 1116.46063 MR 2193205

[4] J. Bellissard, D. J. L. Herrmann, and M. Zarrouati, Hulls of aperiodic solids and gap labeling theorems. In Directions in mathematical quasicrystals, CRM Monogr. Ser. 13, Amer. Math. Soc., Providence, RI, 2000, 207-258. Zbl 0972.52014 MR 1798994

[5] B. Blackadar, K-theory for operator algebras. Math. Sci. Res. Inst. Publ. 5, 2nd ed., Cambridge University Press, Cambridge 1998. Zbl 0913.46054 MR 1656031 
[6] B. Bollobás, Modern graph theory. Grad. Texts in Math. 184, Springer-Verlag, New York 1998. Zbl 0902.05016 MR 1633290

[7] L. E. J. Brouwer, On the stucture of perfect sets of points. Proc. Kon. Nederl. Akad. Wetensch. 12 (1910), 785-794.

http://www.digitallibrary.nl/proceedings/proc_browse.cfm?procid=165

[8] E. Christensen and C. Ivan, Sums of two-dimensional spectral triples. Math. Scand. 100 (2007), 35-60. Zbl 1155.58003 MR 2327719

[9] E. Christensen, C. Ivan, and M. L. Lapidus, Dirac operators and spectral triples for some fractal sets built on curves. Adv. Math. 217 (2008), 42-78. Zbl 1133.28002 MR 2357322

[10] A. Connes, Trace de Dixmier, modules de Fredholm et géométrie riemannienne. Nuclear Phys. B Proc. Suppl. 5B (1988), 65-70. Zbl 0958.58501 MR 1002957

[11] A. Connes, Noncommutative geometry. Academic Press, San Diego, CA, 1994. Zbl 0818.46076 MR 1303779

[12] J. B. Conway, A course in functional analysis. 2nd ed., Grad. Texts in Math. 96, SpringerVerlag, New York 1990. Zbl 0706.46003 MR 1070713

[13] M. Del Muto and A. Figà-Talamanca, Diffusion on locally compact ultrametric spaces. Expo. Math. 22 (2004), 197-211. Zbl 1151.60303 MR 2069670

[14] S. N. Evans, Local fields, Gaussian measures, and Brownian motions. In Topics in probability and Lie groups: boundary theory, CRM Proc. Lecture Notes 28, Amer. Math. Soc., Providence, RI, 2001, 11-50. Zbl 0989.60039 MR 1832433

[15] K. Falconer, Fractal geometry. John Wiley \& Sons Ltd., Chichester 1990. Zbl 0689.28003 MR 1102677

[16] K. Falconer, Techniques in fractal geometry. John Wiley \& Sons Ltd., Chichester 1997. Zbl 0869.28003 MR 1449135

[17] C. Favre and M. Jonsson, The valuative tree. Lecture Notes in Math. 1853, SpringerVerlag, Berlin 2004. Zbl 1064.14024 MR 2097722

[18] A. Figà-Talamanca, Diffusion on compact ultrametric spaces. In Noncompact Lie groups and some of their applications (San Antonio, TX, 1993), NATO Adv. Sci. Inst. Ser. C Math. Phys. Sci. 429, Kluwer Acad. Publ., Dordrecht 1994, 157-167. Zbl 0839.43011 MR 1306522

[19] M. Fukushima, Dirichlet forms and Markov processes. North-Holland Math. Library 23, North-Holland, Amsterdam 1980. Zbl 0422.31007 MR 0569058

[20] J. M. Gracia-Bondía, J. C. Várilly, and H. Figueroa, Elements of noncommutative geometry. Birkhäuser Adv. Texts, Birkhäuser, Boston 2001. Zbl 0958.46039 MR 1789831

[21] D. Guido and T. Isola, Dimensions and singular traces for spectral triples, with applications to fractals. J. Funct. Anal. 203 (2003), 362-400. Zbl 1031.46081 MR 2003353

[22] D. Guido and T. Isola, Dimensions and spectral triples for fractals in $\mathbb{R}^{N}$. In Advances in operator algebras and mathematical physics, Theta Ser. Adv. Math. 5, Theta, Bucharest 2005, 89-108. MR 2238285

[23] F. Hausdorff, Set theory. 2nd ed., Chelsea Publishing Co., New York 1962. MR 0141601 
[24] G.H. Hardy and M. Riesz, The general theory of Dirichlet's series. Cambridge University Press, Cambridge 1915. JFM 45.0387.03 MR 0185094

[25] J. Kigami, Analysis on fractals. Cambridge Tracts in Math. 143, Cambridge University Press, Cambridge 2001. Zbl 0998.28004 MR 1840042

[26] A. N. Kochubei, Pseudo-differential equations and stochastics over non-Archimedean fields. Monographs Textbooks Pure Appl. Math. 244, Marcel Dekker Inc., New York 2001. Zbl 0984.11063 MR 1848777

[27] S. V. Kozyrev, Wavelet theory as p-adic spectral analysis. Izv. Ross. Akad. Nauk Ser. Mat. 66 (2) (2002), 149-158; English transl. Izv. Math. 66 (2002), 367-376. Zbl 1016.42025 MR 1918846

[28] M. L. Lapidus, Towards a noncommutative fractal geometry? Laplacians and volume measures on fractals. In Harmonic analysis and nonlinear differential equations (Riverside, CA, 1995), Contemp. Math. 208, Amer. Math. Soc., Providence, RI, 1997, 211-252. Zbl 0889.58012 MR 1467009

[29] G. Michon, Les cantors réguliers. C. R. Acad. Sci. Paris Sér. I Math. 300 (1985), 673-675. Zbl 0582.54019 MR 802649

[30] K. R. Parthasarathy, Probability measures on metric spaces. Probab. Math. Statist. 3, Academic Press, New York 1967. Zbl 0153.19101 MR 0226684

[31] W. Rudin, Real and complex analysis. 3rd ed., McGraw-Hill, New York 1987. Zbl 0925.00005 MR 0924157

[32] W. H. Schikhof, Ultrametric calculus. Cambridge Stud. Adv. Math. 4, Cambridge University Press, Cambridge 1984. Zbl 0553.26006 MR 0791759

[33] V. S. Vladimirov, I. V. Volovich, and E. I. Zelenov, p-adic analysis and mathematical physics. World Scientific Publishing, Singapore 1994. Zbl 0812.46076 MR 1288093

Received February 10, 2008; revised November 2, 2008

J. Pearson,Georgia Institute of Technology, School of Mathematics, Atlanta,

GA 30332-0160, U.S.A.

E-mail: john.pearson.02@gmail.com

J. Bellissard, Georgia Institute of Technology, School of Mathematics, Atlanta, GA 30332-0160, U.S.A.

E-mail: jeanbel@math.gatech.edu 